\title{
Kritische Herzfehler des Neugeborenen
}

\section{Teil 2: Ausgewählte kritische Herzfehler}

\author{
Martin Böhne, Thomas Jack, Harald Köditz, Philipp Beerbaum
}

\section{Übersicht}

Einleitung

Duktusabhängige Herzfehler

Herzfehler ohne nominelle

Duktusabhängigkeit

\section{Einleitung}

In der letzten Ausgabe haben wir grundlegende Informationen zu kritischen angeborenen Herzfehlern vorgestellt. Der Fokus lag auf der Pathophysiologie, der Diagnostik und den Grundlagen der Behandlung kritischer Herzfehler des Neugeborenen. Auf Basis dieses Wissens werden in diesem Artikel nun ausgewählte kritische Herzfehler des Neugeborenen, deren Pathophysiologie, Diagnostik, initiales Management und die möglichen Therapieoptionen thematisiert.

\section{Duktusabhängige Herzfehler}

Für Vitien mit duktusabhängiger Lungen- oder Systemperfusion gelten für die postnatale Therapie die in der Vorausgabe beschriebenen allgemeinen Therapieprinzipien.

\section{Herzfehler mit duktusabhängiger Lungen- perfusion (Rechtsherzobstruktion)}

\section{Kritische Pulmonalstenose (PS)}

Definition und Epidemiologie. Die isolierte valvuläre

Pulmonalstenose macht etwa 8-12\% aller angeborenen Herzfehler aus. Die kritische Pulmonalstenose des Neugeborenen mit Duktusabhängigkeit ist viel seltener. Hier ist die effektive Klappenöffnungsfläche zu klein, um einen ausreichenden antegraden Fluss zur Lunge und damit eine ausreichende Oxygenierung zu gewährleisten [1].

Anatomie und Hämodynamik. Die Pulmonalklappe ist oft bikuspidal mit teilfusionierten Kommissuren, seltener myxematös verdickt und dysplastisch (typisch bei Noonan-Syndrom). Nicht selten besteht eine begleitende subvalvuläre Stenose des rechtsventrikulären Ausflusstrakts. Schon intrauterin entwickelt sich eine Hypertrophie des rechtsventrikulären Myokards, nicht selten sogar eine konsekutive Hypoplasie des rechten Ventrikels und der Trikuspidalklappe. In dieser Situation kann eine duktusabhängige Lungenperfusion bestehen. Über das Foramen ovale besteht außerdem ein entlastender Rechts-Links-Shunt, der jedoch zusätzlich zur postnatalen Zyanose beiträgt. 


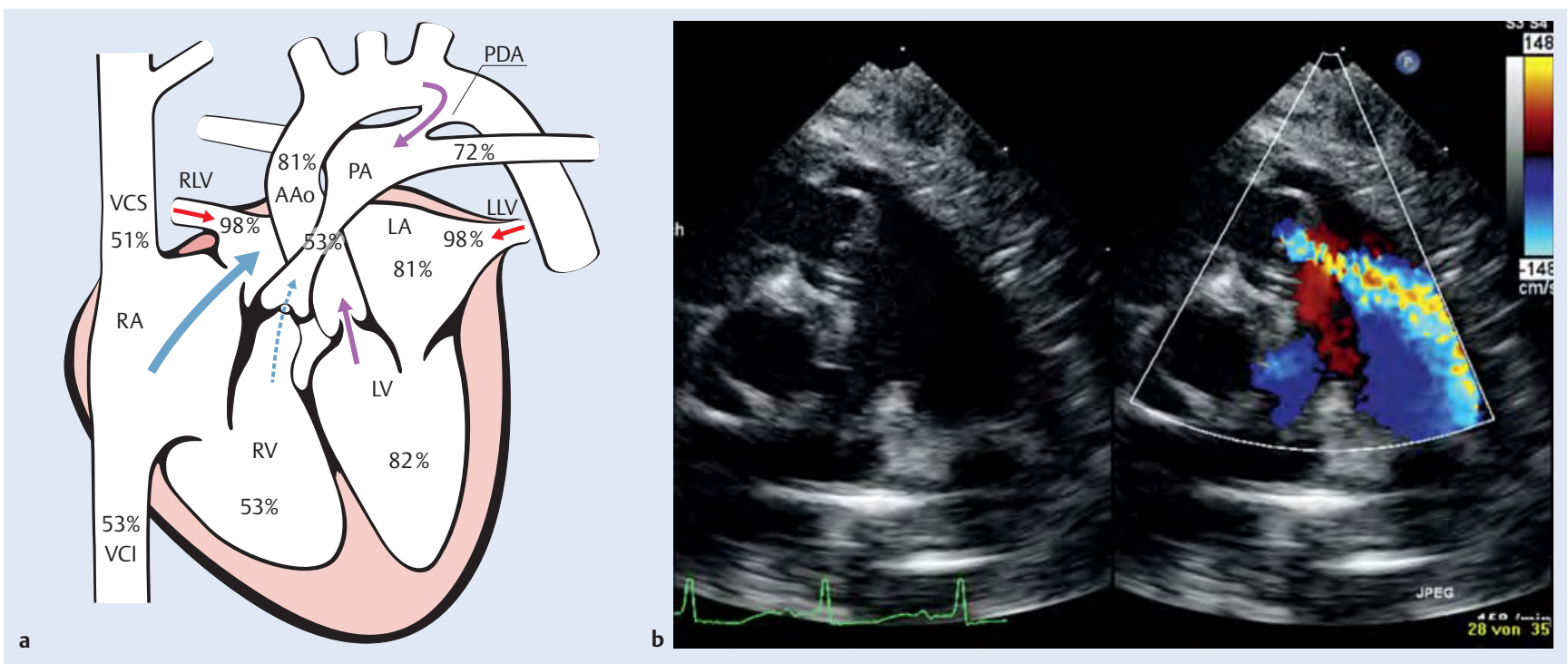

Abb. 1 Kritische Pulmonalstenose des Neonaten. a Grafische Darstellung der Hämodynamik bei kritischer Pulmonalstenose. Angezeigt sind jeweils die Sauer-

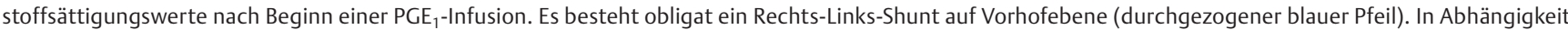
von der Pulmonalstenose, der rechtsventrikulären Funktion und dem Lungengefäßwiderstand findet sich eine unterschiedlich ausgeprägte antegrade Perfusion über die Pulmonalklappe (gestrichelter blauer Pfeil). b Echokardiografische Darstellung in parasternal kurzer Achse am 2. Lebenstag bei einem Zwillingsfrühgeborenen der $32.5 S W$ mit einem Geburtsgewicht von $1700 \mathrm{~g}$. Es zeigt sich eine valvuläre Pulmonalstenose bei dysplastischer Klappenanlage mit einer nur geringen zentralen Öffnungsfläche. Über die Pulmonalklappe stellt sich ein beschleunigter antegrader Fluss mit einer maximalen Geschwindigkeit von $4 \mathrm{~m} / \mathrm{s}$ dar. Der Pulmonalarterienhauptstamm ist poststenotisch dilatiert. Bei nicht ausreichendem antegraden Fluss über die Pulmonalklappe wird der Ductus arteriosus (große Duktusampulle) mittels $\mathrm{PGE}_{1}$-Infusion (Prostaglandin $\mathrm{E}_{1}$ ) zur Sicherstellung der Lungendurchblutung offengehalten. Im Alter von 2 Wochen erhielt das Kind im Herzkatheterlabor eine Ballonvalvuloplastie der Pulmonalklappe. Bei sehr gutem postinterventionellen Ergebnis ohne Reststenose der Pulmonalklappe konnte die $\mathrm{PGE}_{1}$-Infusion beendet werden. Der Dopplergradient über die Pulmonalklappe (Druckdifferenz zwischen rechtem Ventrikel und Pulmonalarterienhauptstamm) kann postnatal durch den noch erhöhten pulmonalarteriellen Widerstand gering sein und damit die Schwere der Pulmonalstenose unterschätzen.

\section{Abkürzungen in Abbildungen}

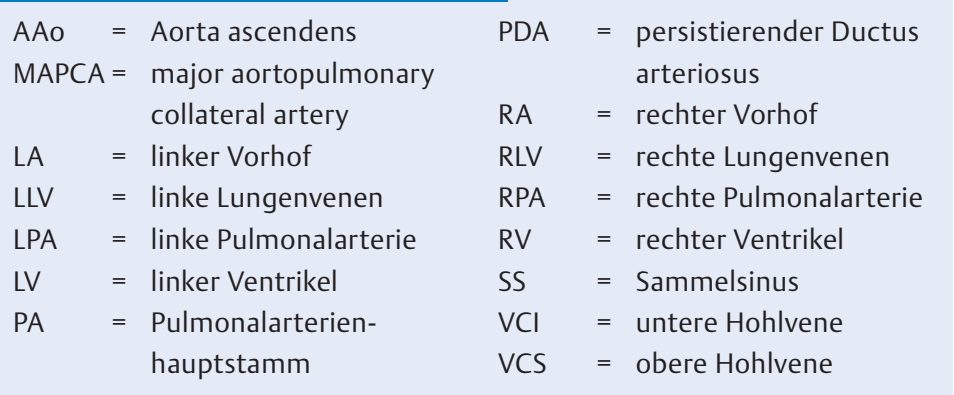

Merke: Für die kritische Pulmonalstenose mit verschlossenem Ductus arteriosus gilt: Je geringer die antegrade Perfusion über die stenotische Pulmonalklappe, desto ausgeprägter ist der Rechts-LinksShunt auf Vorhofebene und damit die Zyanose des Neugeborenen.

Klinik und Diagnostik. Bei Verschluss des Ductus arteriosus präsentieren sich die Neugeborenen mit:

- Zyanose

- Tachypnoe

- Zeichen der Rechtsherzdekompensation mit Hepatomegalie
Ein gespaltener 2. Herzton sowie ein raues, spindelförmiges Systolikum mit Punctum maximum im 2./3.ICR links parasternal und Fortleitung in beide Thoraxhälften ist auskultierbar. Bei rechtsventrikulärer Dekompensation kann das Austreibungsgeräusch komplett fehlen. Im Röntgen-Thorax zeigt sich eine verminderte Lungengefäßzeichnung, echokardiografisch stellt sich eine verdickte, kaum öffnende Pulmonalklappe mit nur einem geringen antegraden Fluss, ein Rechts-LinksShunt über das Foramen ovale und ein Links-RechtsShunt bei offenem Ductus arteriosus dar (Abb.1).

Merke: Echokardiografisch korreliert der Gradient (z. B. CW-Doppler) über die Pulmonalklappe nicht immer mit dem Schweregrad einer Pulmonalklappenstenose: Die Flussgeschwindigkeit über die stenosierte Klappe ist reduziert, wenn bereits eine eingeschränkte rechtsventrikuläre Pumpfunktion besteht und/oder wenn der Lungengefäßwiderstand noch erhöht ist.

Management. Bei zentraler Zyanose ist eine $\mathrm{PGE}_{1}$-Infusion zum Offenhalten bzw. Wiedereröffnen des Ductus arteriosus indiziert. Nach Stabilisierung erfolgt als Therapie der Wahl eine katheterinterventionelle Ballonvalvuloplastie [2]. Diese führt in über $90 \%$ der Fälle zu 

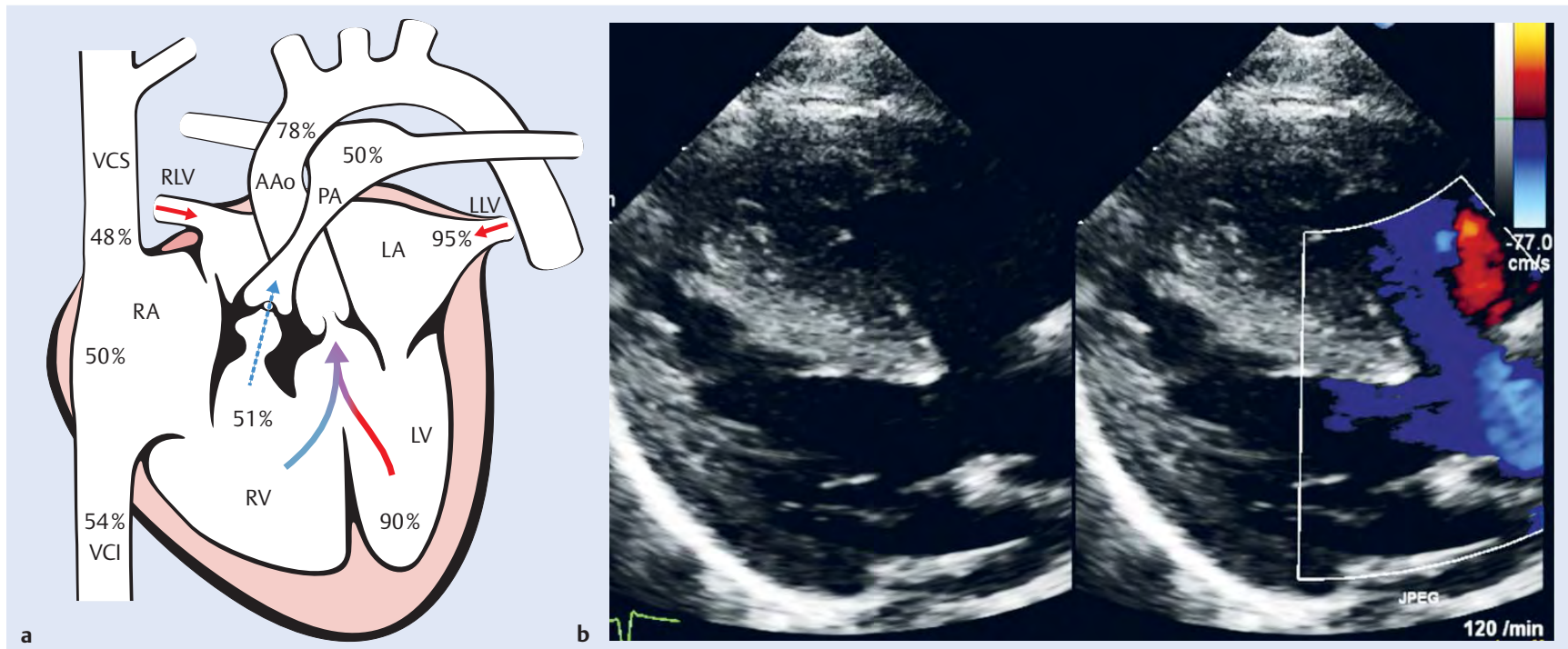

Abb. 2 Hämodynamik eines Patienten mit Fallot-Tetralogie. a Sauerstoffsättigungswerte bei Fallot-Tetralogie mit infundibulärer und valvulärer Pulmonalstenose. Der blaue Pfeil stellt den intrakardialen Rechts-Links-Shunt über den VSD dar. Aufgrund der Obstruktion im rechtsventrikulären Ausflusstrakt ist der antegrade Fluss in die Lungenarterien reduziert (gestrichelter blauer Pfeil über die Pulmonalklappe). b Echokardiografie (parasternal lange Achse) eines Patienten mit Fallot-Tetralogie. Darstellung eines perimembranösen Malalignment-VSD. Die Aortenwurzel überreitet den VSD. Im Farbdoppler (rechts): In der Systole Darstellung eines Rechts-Links-Shunts über den VSD mit Ausstrom des rechtsventrikulären Blutes über die Aortenklappe.

einer Reduktion des Druckgradienten. Bei einigen Neugeborenen ist die antegrade Perfusion der Lungen über die Pulmonalklappe nach der Ballonvalvuloplastie aufgrund einer rechtsventrikulären Hypoplasie noch nicht ausreichend. Diese Kinder benötigen zum mittelfristigen Offenhalten des Ductus arteriosus entweder eine Fortsetzung der Prostaglandin- $\mathrm{E}_{1}$-Infusion $\left(\mathrm{PGE}_{1}{ }^{-}\right.$ Infusion) für einige Zeit oder - falls anatomisch möglich - eine Stent-Implantation in den Ductus arteriosus. Alternativ kann die chirurgische Anlage eines aortopulmonalen Shunts bei Persistenz der Duktusabhängigkeit notwendig sein.

Ca. 15\% der Patienten benötigen im späteren Alter eine Reintervention an der Pulmonalklappe.

Bei schwerer Klappendysplasie (z. B. beim NoonanSyndrom) sind die Ergebnisse der Ballonvalvuloplastie schlechter (Erfolgsrate maximal 60-65\%), ein Versuch ist jedoch meist gerechtfertigt. Nicht selten besteht in dieser Konstellation eine zusätzliche supravalvuläre Pulmonalarterienstenose am sinutubulären Übergang.

\section{- Fallot-Tetralogie (TOF)}

Definition und Epidemiologie. Die Fallot-Tetralogie (TOF) ist der häufigste zyanotische Herzfehler (ca. 5-10\% aller angeborenen Herzfehler; AHF) [1,3]. Er ist nicht selten mit einer Mikrodeletion 22q11 assoziiert. Der Herzfehler ist charakterisiert durch eine Deviation des Konusseptums nach rechts-anterior mit konse- kutiver rechtsventrikulärer Ausflusstraktobstruktion (valvuläre, subvalvuläre und oft auch supravalvuläre Pulmonalstenose) mit resultierender rechtsventrikulärer Hypertrophie, einem großen „Malalignment“Ventrikelseptumdefekt (VSD) und einer über dem VSD reitenden, entsprechend der Position des Konusseptums ebenfalls nach rechts-anterior verlagerten Aortenwurzel (Abb.2a).

\section{Anatomie und Hämodynamik}

Merke: Das Ausmaß der Obstruktion des rechtsventrikulären Ausflusstrakts bestimmt den intrakardialen Rechts-Links-Shunt und damit die Zyanose. Aufgrund des großen VSD besteht ein identischer Druck in beiden Ventrikeln. Die Aorta ist durch ihr Überreiten des VSD funktionell beiden Ventrikeln zugeordnet.

Die Ausprägung der rechtsventrikulären Ausflussobstruktion bestimmt die Klinik des Neugeborenen:

- Bei nur geringer Ausprägung der rechtsventrikulären Ausflusstraktobstruktion fließt ausreichend Blut in den Lungenkreislauf und die Neugeborenen sind postnatal azyanotisch („Pink Fallot“). Normalerweise nimmt die Ausprägung der rechtsventrikulären Ausflusstraktobstruktion innerhalb der ersten Monate zu. Der Pulmonalklappenring wie auch der Pulmonalarterienhauptstamm sind häufig hypoplastisch, die Pulmonalarterien schmal. In einigen Fällen erfolgt die Lungenperfusion zusätzlich über aortopulmonale 


\section{Übersicht hypoxämischer Anfall (kinderkardiologischer Notfall)}

Hämodynamik

akuter Abfall des peripheren Widerstands und/oder Obstruktion des rechtsventrikulären Ausflusstrakts

\section{Typische Auslöser}

körperliche oder psychische Belastung (Schreien, Aufwachen), Abfall des Systemwiderstands (Füttern, Baden), Hypovolämie, Fieber

\section{Klinik}

- initial Zunahme der Zyanose mit Tachykardie und Tachypnoe, gefolgt von Zeichen der Systemkreislaufinsuffizienz mit blass-grauem Hautkolorit, Tonusverlust der Muskulatur, Bewusstseinsverlust, zerebrale Krampfanfälle („,blasses oder zyanotisches Kind ohne adäquate Reaktion")

- kein Ausströmungsgeräusch auskultierbar

\section{Therapie}

- Erhöhung des systemischen Widerstands zur Steigerung der antegraden Perfusion in die Pulmonalarterien (Knie an die Brust beugen, Hockstellung)

- großzügige intravenöse Volumengabe (mehrfache Bolusgaben von $10 \mathrm{ml} / \mathrm{kg} \mathrm{KG}$ )
- Sedierung (Morphin 0,1-0,2 mg/kg KC s. c., i.v., i. m.; Ketamin 1-2 mg/kg KG i.v.)

- Sauerstoffgabe

- Ausgleich einer metabolischen Azidose durch Pufferung mit Natriumbikarbonat

- bei Persistenz der Zyanose intensivmedizinische Therapie mit intravenöser Applikation von Vasokonstriktoren (Noradrenalin), ß-Blockern

\section{Prophylaxe}

ß-Blocker (z. B. Propranolol

2-6 mg/kg KG p.o. in 3-4 ED)
Kollateralen („major aortopulmonary collateral arteries“, MAPCAs), die ehemaligen embryonalen Gefäßen zwischen Lunge und Aorta entsprechen.

- Bei einer ausgeprägten rechtsventrikulären Ausflusstraktobstruktion fließt ein großer Anteil des desoxigenierten Blutes, wie in Abb. 2 dargestellt, aufgrund des hohen Auswurfwiderstands anstelle in den Lungenkreislauf über die Aorta in den Körperkreislauf (intrakardialer Rechts-Links-Shunt; „Blue Fallot“).

Merke: Bei einer ausgeprägten Stenosierung des rechtsventrikulären Ausflusstrakts (funktionelle Pulmonalatresie) können Neugeborene bereits direkt postnatal mit einer Zyanose auffällig werden.

- Im Extremfall tritt eine Atresie der Pulmonalklappe auf. Diese kommt in ca. 7\% der Patienten mit TOF vor [1]. Bei diesen Patienten sind ein offener Ductus arteriosus oder MAPCAs für die Oxygenierung notwendig (siehe auch nachfolgender Abschnitt über Pulmonalatresie mit VSD).

In $15-20 \%$ besteht ein rechter Aortenbogen, nicht selten finden sich auch Ursprungsanomalien der Koronararterien, die ggf. für die operative Korrektur relevant sein können. Sehr selten zeigt sich ein doppelter Aortenbogen.

Klinik und Diagnostik. Bei Verschluss des Ductus arteriosus fallen Neugeborene mit funktioneller oder anatomischer Pulmonalatresie durch eine Zyanose auf.
Merke: In den meisten Fällen ist die Zyanose im Neugeborenenalter nur gering ausgeprägt (sog. Pink Fallot) und wird erst nach einigen Monaten offensichtlich, entsprechend besteht keine neonatale Duktusabhängigkeit der Lungenperfusion.

Ein 3/6-gradiges raues spindelförmiges Systolikum mit p.m. über 2./3. ICR links parasternal und thorakaler Fortleitung wird durch die Obstruktion der rechtsventrikulären Ausflussbahn verursacht. Das EKG zeigt unspezifisch eine Rechtshypertrophie (Abb. 10 der Vorausgabe). Die Echokardiografie sichert die Diagnose, stellt kardiale Begleitfehlbildungen und die Ausprägung der strukturellen Anomalie bzw. ihre funktionelle Relevanz dar. Das Röntgenbild des Thorax zeigt die typische Herzsilhouette („Golfschlägerherz“) und ermöglicht die Beurteilung der verminderten pulmonalen Gefäßzeichnung als Hinweis auf eine mögliche Hypoplasie des pulmonalarteriellen Systems.

Merke: Bei sehr hypoplastischer Lungenstrombahn und/oder Verdacht auf multifokale Lungenperfusion und/oder assoziierten Koronararterienanomalien ist eine Herzkatheteruntersuchung oder eine CT-Angiografie erforderlich.

Management. In der Mehrzahl der Fälle ist bei einem „Pink Fallot“ in der frühen Neonatalperiode keine spezifische Therapie notwendig.

Als Standardtherapie wird eine Korrekturoperation mit Erweiterung des rechtsventrikulären Ausflusstrakts und VSD-Verschluss im 3.-12. Lebensmonat durchgeführt, entweder primär bei Pink Fallot oder sekundär 
nach vorheriger Palliation (s. u.) bei initialer Duktusabhängigkeit oder bei rascherer Progression der Zyanose im jungen Säuglingsalter.

Bei neonataler zentraler Zyanose und duktusabhängiger Lungenperfusion ist zunächst eine passagere $\mathrm{PGE}_{1}$ Infusion notwendig. Zur invasiven Palliation vor der eigentlichen Korrekturoperation gibt es je nach individuellen anatomischen Gegebenheiten und Erfahrung des Zentrums verschiedene Optionen:

- Katheterinterventionell kann ein Stent in den Ductus arteriosus implantiert werden, falls die Duktusanatomie dieses zulässt.

- Katheterinterventionell kann eine Ballondilatation der Pulmonalklappe erfolgen, wenn diese Komponente überwiegt.

- Katheterinterventionell kann -in entsprechend erfahrenen Händen - eine Stentimplantation über die rechtsventrikuläre Ausflussbahnstenose und Pulmonalklappenstenose erwogen werden.

- Ebenso kann die ausreichende Lungenperfusion klassischerweise durch einen chirurgischen aortopulmonalen Shunt sichergestellt werden.

- Auch kann eine operative Frühkorrektur angestrebt werden.

Eine primäre Korrektur-OP ist durchaus bereits im Neugeborenenalter möglich, geht jedoch dann mit einer erhöhten perioperativen Mortalität und oft weniger günstigem hämodynamischen Resultat einher [3]. Daher bevorzugen die meisten Zentren noch immer ein zweizeitiges Vorgehen, wenn bereits im Neugeborenenalter eine Behandlungspflicht besteht.

Auch können hypoxämische Anfälle bereits in der Neonatalperiode auftreten, normalerweise präsentieren sie sich aber ab dem 3. -4. Lebensmonat.

Merke: Das Auftreten von hypoxämischen Anfällen ist ein kinderkardiologischer Notfall. Nach der Akutbehandlung (siehe Infobox Übersicht hypoxämischer Anfall) ist eine definitive Sicherung der Lungenperfusion notwendig, entweder als Korrektur-OP oder bei jüngeren Säuglingen mittels Palliation.

\section{Pulmonalatresie mit VSD (PA-VSD)}

Definition und Epidemiologie. Bei der PA-VSD (ca. 1\% aller AHF) ist im Gegensatz zur Pulmonalatresie mit intaktem Ventrikelseptum (PA-IVS) der rechte Ventrikel meist gut entwickelt [4]. Der Herzfehler entspricht damit einer Extremvariante der Fallot-Tetralogie mit

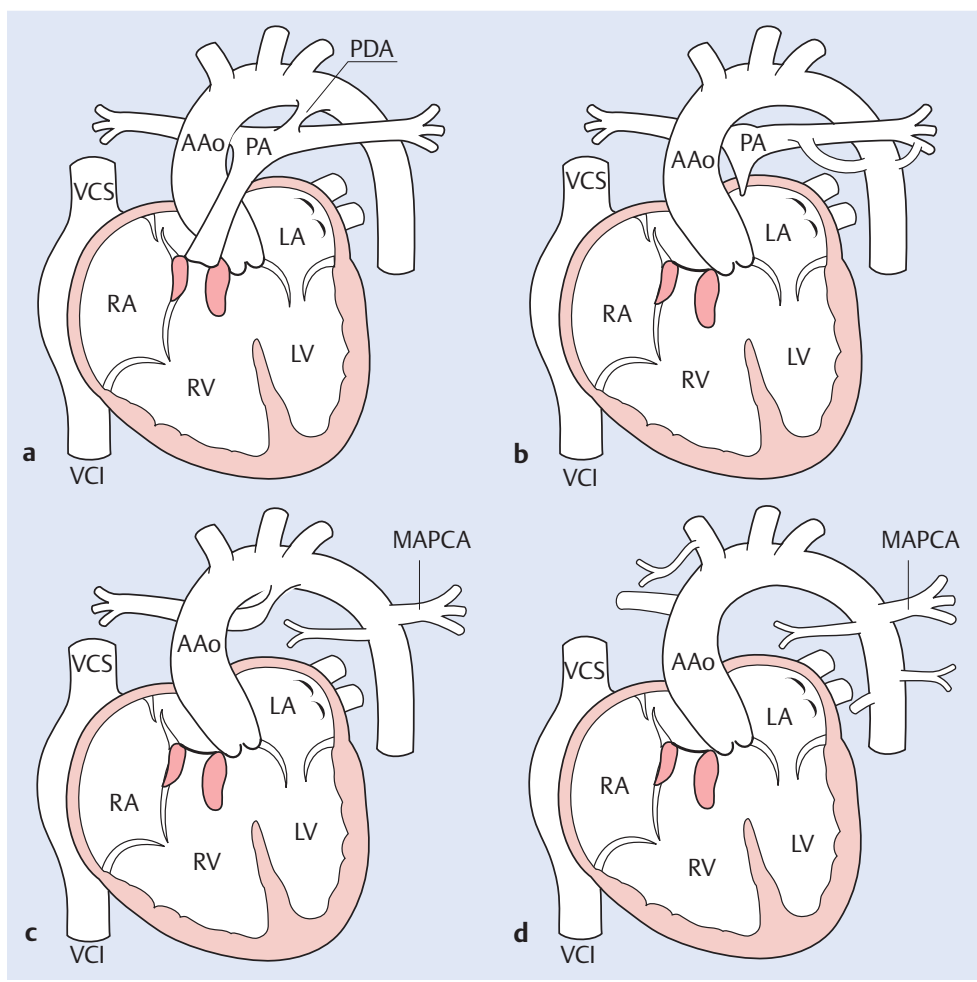

Abb. 3 Einteilung der Pulmonalatresie mit Ventrikelseptumdefekt. Verschiedene morphologische Varianten einer Pulmonalatresie mit Ventrikelseptumdefekt existieren, die sich hauptsächlich in der Ausprägung der Hypoplasie der Lungenarterien und dem Vorhandensein von „major aortopulmonary collateral arteries“ (MAPCAs) unterscheiden. a Zentrale und periphere Pulmonalarterien angelegt, Ductus arteriosus vorhanden. b Rechte und linke zentrale Pulmonalarterie konfluierend angelegt, kein Pulmonalarterienhauptstamm, Ductus arteriosus und/oder MAPCAs vorhanden. c Rechte und linke zentrale Pulmonalarterie aplastisch oder hypoplastisch, kein Pulmonalarterienhauptstamm, kein Ductus arteriosus, MAPCAs vorhanden. d Keine zentralen Pulmonalarterien, Lungendurchblutung ausschließlich über MAPCAs, multifokal.

einer Atresie der Pulmonalklappe. Dieser Herzfehler ist gehäuft mit einer Mikrodeletion 22q11 assoziiert.

Anatomie und Hämodynamik. Die wesentliche morphologische und funktionelle Variable ist die Ausbildung der Lungenarterien, hier gibt es ein weites Spektrum (Abb.3). Die Perfusion der hypoplastischen Lungenarterien erfolgt entweder unifokal (ein zentrales zuführendes Gefäß) über einen Ductus arteriosus oder multifokal über sog. „major aortopulmonary collateral arteries“ (MAPCAs), die residuale embryonale Gefäße zwischen Lunge und Aorta darstellen. In der Extremform können die zentralen Pulmonalgefäße vollständig fehlen, dann erfolgt die Lungenperfusion ausschließlich über MAPCAs und es besteht keine Duktusabhängigkeit (Abb.3d). Ferner gibt es:

- Varianten mit fehlenden zentralen Lungenarterien

- Verzweigungsanomalien

- Stenosen der peripheren Lungenarterien

- duale Versorgung der Lunge, sowohl durch hypoplastische Lungenarterien (duktusabhängig!) als auch 
MAPCAs, ggf. liegen auch Stenosierungen dieser Gefäße vor.

Klinik und Diagnostik. Neugeborene mit PA-VSD fallen mit einer Zyanose auf, deren Ausprägung von der Ausbildung von MAPCAs abhängig ist. Ein systolisches Austreibungsherzgeräusch fehlt, im Unterschied zur FallotTetralogie. Eventuell ist ein systolisch-diastolisches Herzgeräusch als Hinweis auf einen Ductus arteriosus oder aortopulmonale Kollateralarterien zu hören. Die Diagnose wird durch die Echokardiografie gestellt. Hier zeigen sich ein verschlossener rechtsventrikulärer Ausflusstrakt sowie eine über einem VSD überreitende Aorta. Eine diagnostische Herzkatheteruntersuchung bzw. CT-Angiografie zur Darstellung der Anatomie und Perfusion der Lungenarterien ist notwendig. Hierdurch sollte evaluiert werden:

- Perfusion der Lungenarterien

- Vorhandensein von zentralen Lungenarterien

- Vorhandensein einer Bifurkation

- Vorhandensein eines Ductus arteriosus

- MAPCAs

Management. Bei duktusabhängiger Lungenperfusion (keine MAPCAs, zentrale Pulmonalarterien sind angelegt) erfolgt die Applikation einer $\mathrm{PGE}_{1}$-Infusion.

Merke: Bei der Pulmonalatresie mit VSD ist eine Darstellung der Perfusion der Lungenarterien (Duktusabhängigkeit vs. MAPCAs) notwendig. Eventuell besteht trotz atretischer Pulmonalklappe keine $\mathrm{PGE}_{1}$-Abhängigkeit.

Der weitere Behandlungsplan ist häufig individuell und hängt entscheidend von der Anatomie der arteriellen Lungengefäßversorgung ab. Er umfasst neben operativen Maßnahmen zur Unifokalisation etwaiger MAPCAs mit deren Anbindung an die zentralen Pulmonalarterien auch katheterinterventionelle Therapien wie die Coil-Embolisationen von MAPCAs. Ziel ist die Herstellung einer aufnahmefähigen rekonstruierten zentralen Pulmonalarterie und deren Anbindung an den rechten Ventrikel über eine Gefäßprothese (Konduit). Bei günstiger Anatomie kann dabei auch der VSD durch einen Patch verschlossen werden, bei sehr hypoplastischen Pulmonalgefäßen wird man erst deren Wachstum und (interventionelle) Therapie abwarten wollen, um den rechten Ventrikel vor einer zu hohen, suprasystemischen Druckbelastung zu schützen.

\section{- Pulmonalatresie mit intaktem Ventrikelseptum (PA-IVS)}

Definition und Epidemiologie. Bei der PA-IVS (<1\% aller AHF, ca. 2,5\% der kritischen AHF) gelangt das Blut des rechten Ventrikels nicht über die atretische Pulmonalklappe (muskulär-infundibuläre oder valvulär-membranöse Atresie) in die Pulmonalarterien [1].

Anatomie und Hämodynamik. Die fetale Zirkulation ist im ersten Teil in Abb. 4 dargestellt. Die Pulmonalarterien werden über den Ductus arteriosus retrograd durchblutet (wichtiger Marker in der fetalen Echokardiografie!). Das rechtsventrikuläre Blut entleert sich z.T. unter hohem Druck entweder über die insuffiziente Trikuspidalklappe in den rechten Vorhof oder über Fisteln (sog. Myokardsinusoide), die mit den Koronararterien in Verbindung stehen. Die Koronararterien können stenosiert oder atretisch sein. Zudem kann die Koronarperfusion über sinusoidale Verbindungen vom rechten Ventrikel abhängig sein. Normalerweise besteht der rechte Ventrikel aus drei Anteilen, einem Einlass-(Inlet-), einem trabekularisierten und einem Auslass-(Outlet-) Teil.

Merke: Bei der PA-IVS weisen Größe und Morphologie des rechten Ventrikels und der Trikuspidal-

klappe eine große Variabilität auf.

Das Spektrum reicht von einer ausgeprägten Hypoplasie der rechten Kammer und Trikuspidalklappe mit einer fast vollständigen Lumenobliteration bis hin zu einer nur milden Hypoplasie. Der Blutdruck im rechten Ventrikel ist höher als im linken Ventrikel („suprasystemisch“).

Postnatal hängt die Oxygenierung vom Blutfluss über den offenen Ductus arteriosus in die Lungengefäße ab. Das Vorhofseptum ist normalerweise nicht restriktiv mit obligatem (!) Rechts-Links-Shunt (d.h. der gesamte systemvenöse Rückstrom muss durch das Vorhofseptum zur linken Herzseite passieren). Bei Verschluss des Ductus arteriosus bricht die Lungenperfusion zusammen und die Neugeborenen präsentieren sich mit einer schweren Zyanose und sekundärem Kreislaufversagen.

Klinik und Diagnostik. Es besteht eine zentrale Zyanose bei duktusabhängiger Lungenperfusion. Echokardiografisch (Abb.4) zeigen sich neben einem breiten Spektrum in der Morphologie des rechten Ventrikels und der Trikuspidalklappe zusätzlich zur Atresie der Pulmonalklappe (valvulär oder infundibulär) ggf. auch Koronarfisteln. Wichtig ist ferner die Beurteilung des 

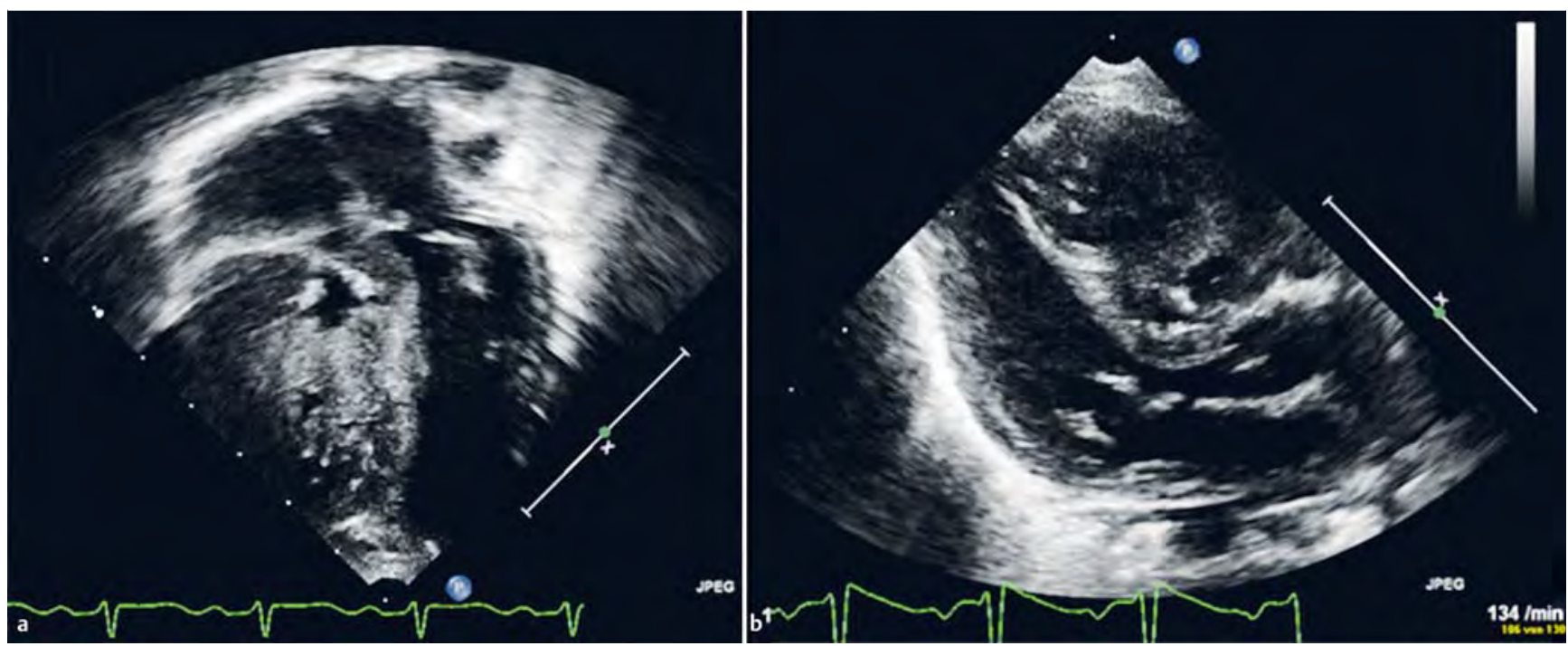

Abb.4 Echokardiografie eines Patienten mit Pulmonalatresie und intaktem Ventrikelseptum. a Im Vierkammerblick zeigt sich deutlich die Myokardhypertrophie des rechten Ventrikels bei nur kleinem Cavum. b Korrespondierende parasternal lange Achse.

Vorhofseptums: Hier besteht ein obligater RechtsLinks-Shunt, eine Restriktion ist eher selten.

Merke: Für die weitere individualisierte Therapieplanung bei großer anatomischer Variabilität und zur Beurteilung der Koronarperfusion ist in der Regel eine diagnostische Herzkatheteruntersuchung notwendig.

Sollte das Vorhofseptum restriktiv sein (indirekte Zeichen: Hepatomegalie und Lebervenen-/Hohlvenenstauung durch Rückstau!), kann eine Ballonatrioseptostomie nach Rashkind angezeigt sein (s. Kapitel „Transposition der großen Arterien“).

Merke: Solange sowohl die Inlet- als auch die Outlet-Portion des rechten Ventrikels angelegt sind, kann durch geeignete palliative Zwischenschritte ein Wachstum induziert werden, welches nicht selten nach einigen Jahren eine Zweikammer-Korrektur erlaubt. Dennoch verbleibt meist eine restriktive Funktionsstörung des rechten Ventrikels [5].

Management. Der sofortige Beginn einer $\mathrm{PGE}_{1}$-Infusion und entsprechender supportiver Therapiemaßnahmen (s. Teil 1 in der Vorausgabe) ist indiziert. Die individuelle Therapieplanung hängt von der Morphologie des rechten Ventrikels, der Größe der Trikuspidalklappe und der Existenz von signifikanten Koronarfisteln oder -anomalien ab:

- Bei Vorliegen einer membranösen Pulmonalatresie und ausreichend großem rechten Ventrikel und ausreichender Trikuspidalklappe kann die Ausflussbahn zu den Pulmonalarterien katheterinterventionell oder chirurgisch in der Neonatalperiode eröffnet werden, um eine Dekompression und damit ein weiteres Größenwachstum des rechten Ventrikels zu erzielen [6,7]. In diesem Fall ist meist ein „tripartiter“ (dreiteiliger) rechter Ventrikel vorhanden und die Trikuspidalklappe ausreichend groß, entsprechend wird eine biventrikuläre Korrektur angestrebt.

- In weniger günstigen Fällen ist eine teilentlastende Operation mit einem Anschluss der oberen Hohlvene an die Pulmonalarterien (obere cavopulmonale Anastomose [CPA]) und PFO-/ASD-Verschluss möglich (Abb. 11). Die Pulmonalarterie wird jedoch nicht abgesetzt. Damit erreicht das systemvenöse Blut die Lungenarterien über zwei Wege: über die CPA aus der oberen Hohlvene und antegrad über die Pulmonalklappe aus der unteren Hohlvene über den rechten Ventrikel. Der rechte Ventrikel ist somit volumenentlastet, da er nur das Blut der unteren Hohlvene über die eröffnete Pulmonalklappe pumpen muss (sog. „one-and-a-half-repair").

- Ist der rechte Ventrikel stark hypoplastisch mit extrem hypoplastischer Trikuspidalklappe und/oder einer vom rechten Ventrikel abhängigen Koronarperfusion, kann zur Kreislauftrennung letztendlich nur eine stufenweise univentrikuläre Palliation erfolgen: - Zunächst wird die Lungenperfusion durch einen chirurgischen aortoplumonalen Shunt oder einen Duktusstent gesichert.

- Danach erfolgt mit 4-6 Monaten ein Anschluss der oberen Hohlvene an die Pulmanalarterien (obere CPA). Hierbei wird der Pulmonalarterienstamm vom Herzen abgesetzt (s. Abb.11). 


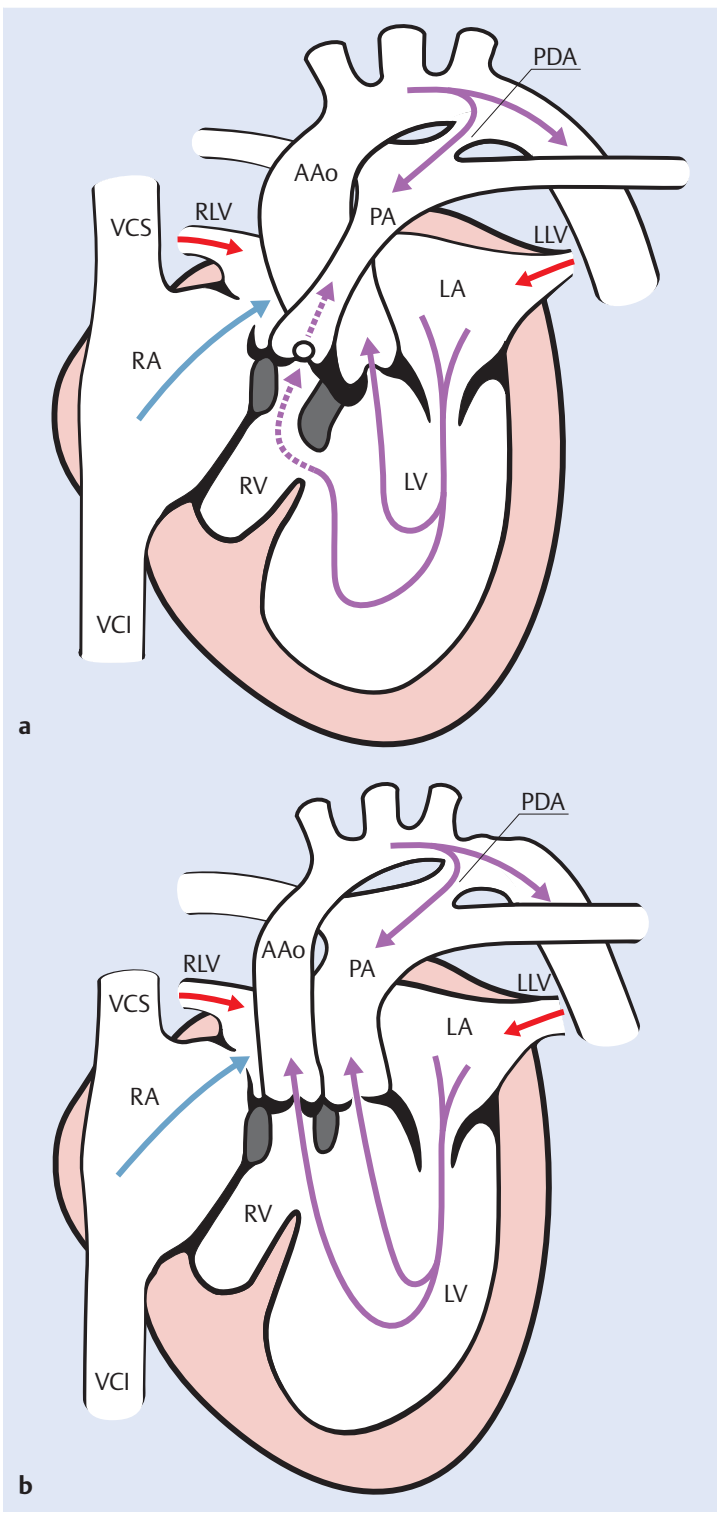

- Mit etwa 2 Jahren folgt schließlich die Komplettierung der Kreislauftrennung nach dem Fontanprinzip durch Anschluss auch der unteren Hohlvene an die rechte Pulmonalarterie (intrakardialer oder extrakardialer Tunnel), inklusive Fenestration des Tunnels zum pulmonalvenösen Vorhof und Atrioseptektomie (s. Abb.12).

Merke: Bei einer vom rechten Ventrikel abhängigen Koronarperfusion ist eine Dekompression des rechten Ventrikels kontraindiziert, da ansonsten eine Flussumkehr in den Koronarien mit einem Abfluss in den rechten Ventrikel mit konsekutiver Myokardischämie resultieren würde. Hier bleibt nur die Fontanzirkulation. Die Gesamtprognose ist eingeschränkt.
Abb. 5 Morphologische Varianten der Trikuspidalatresie. Die schematischen Abbildungen zeigen die beiden häufigsten Varianten der Trikuspidalatresie. In der Mehrzahl findet sich eine Normalstellung der großen Arterien (a), in ca. einem Drittel der Fälle auch eine Transposition der großen Arterien (TGA) mit Ursprung der Aorta aus dem hypoplastischen rechten Ventrikel und der Lungenarterien aus dem linken Ventrikel (b). Immer findet sich ein obligater Rechts-Links-Shunt auf Vorhofebene (blauer Pfeil). Die Aorta wie auch die Pulmonalarterie erhalten nach Mischung des desoxigenierten systemvenösen (blauer Pfeil) mit dem volloxigenierten pulmonalvenösen Blut (rote Pfeile) im linken Vorhof und Ventrikel beide jeweils Blut mit reduzierter Sauerstoffsättigung (violette Pfeile). Dabei bestimmt das Ausmaß der Lungenperfusion die Höhe der Sauerstoffsättigung.

Die Abb. a zeigt eine Trikuspidalatresie Typ lb (häufgste Variante) mit einer Normalstellung der großen Arterien. Hier finden sich häufig ein restriktiver Ventrikelseptumdefekt und eine zusätzliche Pulmonalstenose, die den Blutfluss zur Lunge vermindern (gestrichelte violette Pfeile). Die Lungenarterien sind in der Regel hypoplastisch. Die Patienten fallen mit einer Zyanose auf. Ggf. ist die pulmonale Perfusion duktusabhängig. b Trikuspidalatresie Typ Ilc mit einer TGA. Liegt keine Pulmonalstenose vor, ist die Lungendurchblutung vermehrt und die Kinder sind nur gering zyanotisch und fallen durch klinische Zeichen der Herzinsuffizienz auf. Die Aorta wird über den VSD perfundiert. Bei großem VSD kann die Aorta normal groß sein, bei kleinem VSD oder einer subaortalen Stenose besteht häufig eine Aortenbogenanomalie (Hypoplasie der Aorta ascendens, des Aortenbogens oder ISTA). Im Extremfall kann die Perfusion des Systemkreislaufs auch duktusabhängig sein.

\section{Trikuspidalatresie (mit und ohne d-Transposition der großen Arterien)}

Definition und Epidemiologie. Die Trikuspidalatresie ist nach der Fallot-Tetralogie und der Transposition der großen Arterien der dritthäufigste zyanotische Herzfehler (ca. 1-3\% aller AHF). Sie ist durch eine rudimentäre oder (meist) vollständig fehlende Anlage der Trikuspidalklappe und daraus folgend einem fehlenden Einlassteil des rechten Ventrikels gekennzeichnet.

Anatomie und Hämodynamik. Zwischen dem rechten Vorhof und Ventrikel existiert keine direkte Verbindung (Abb.5). Das systemvenöse Blut gelangt vom rechten Vorhof durch eine Lücke im Vorhofseptum in den linken Vorhof (obligater Rechts-Links-Shunt). Der rechte Vorhof ist meist dilatiert. Da sowohl das gesamte systemvenöse als auch das pulmonalvenöse Blut durch den linken Vorhof und Ventrikel strömen, dilatieren auch diese. In ca. $90 \%$ besteht ein Ventrikelseptumdefekt (VSD) [5], der den Zufluss zum rechten Ventrikel sicherstellt und mit dessen Größe korreliert. Im linken Ventrikel findet eine komplette Durchmischung des systemvenösen und pulmonalvenösen Blutes statt. Von hier aus strömt es mit einer identischen Sättigung in die Aorta und die Pulmonalarterie. Die Flussrate in beiden großen Arterien hängt von verschiedenen Faktoren ab: 
- Bei ca. zwei Drittel der Fälle besteht eine ventrikuloarterielle Konkordanz, d. h. die großen Arterien entspringen regelrecht aus den Ventrikeln (Aorta aus linkem Ventrikel, Pulmonalis aus rechtem Ventrikel, Abb.5a). Hier ist der VSD meist klein und der rechte Ventrikel hypoplastisch. Ein restriktiver VSD vermindert die Lungenperfusion und damit die effektive Sauerstoffaufnahme, es resultiert eine ausgeprägte zentrale Zyanose .

- Bei ca. einem Drittel der Fälle besteht eine ventrikuloarterielle Diskordanz, d.h. es liegt zusätzlich eine Transpositionsstellung der großen Arterien vor (Abb.5b). Die Aorta entspringt aus dem hypoplastischen rechten Ventrikel, der sein Blut ausschließlich über einen VSD erhält, die Pulmonalarterie dagegen aus dem linken Ventrikel. Entsprechend wird der VSD für die Durchblutung des Systemkreislaufs über die Aorta benötigt. Die Größe der Aorta korreliert mit der Größe des VSD, d. h. bei einem großen VSD kann die Aorta normal groß sein, bei einem kleinen VSD ist diese oft hypoplastisch, nicht selten mit zusätzlicher Aortenbogenhypoplasie und Aortenisthmusstenose (ISTA) [4], mit Übergangsformen zur duktusabhängigen Systemperfusion. Der pulmonale Blutfluss erfolgt über die Pulmonalarterie aus dem linken Ventrikel und ist nach dem postnatal physiologischen Abfall des Lungengefäßwiderstands oft gesteigert. In diesem Fall weisen die betroffenen Neugeborenen eine pulmonale Hyperperfusion mit geringer Zyanose auf, oft entwickelt sich eine Herzinsuffizienz.

Merke: Bei Trikuspidalatresie und normaler Gefäßrelation kann eine duktusabhängige Lungenperfusion bestehen. Bei Trikuspidalatresie mit Transposition der großen Arterien kann dagegen eine duktusabhängige Systemperfusion bestehen.

Neben der Größe des VSD und der Stellung der großen Arterien wird der pulmonale Blutfluss auch noch von der Existenz einer Pulmonalstenose (ca. ein Drittel der Fälle) bestimmt. Bei ausgeprägter Pulmonalstenose oder -atresie ist der pulmonale Blutfluss noch weiter reduziert und die Lungenperfusion ggf. duktusabhängig. Oft ist eine Trikuspidalatresie mit weiteren Fehlbildungen (ISTA, unterbrochener Aortenbogen) assoziiert, insbesondere bei gleichzeitiger Transposition der großen Arterien.
Merke: Bei der Trikuspidalatresie bestimmt der pulmonale Blutfluss die Sättigung. Dieser hängt von vielen Faktoren ab: Ursprung der großen Arterien aus den Ventrikeln, Dimension eines VSD, Vorhandensein einer Pulmonalstenose, Durchmesser eines Ductus arteriosus und relativer Widerstand der Lungengefäße.

Klinik und Diagnostik. Klinisch fallen die Neugeborenen durch eine unterschiedlich ausgeprägte Zyanose auf. Die Klinik reicht von tiefer Zyanose bei duktusabhängiger Lungenperfusion bis zu VSD-typischer Herzinsuffizienz mit nur geringer Zyanose. Man auskultiert meist einen singulären 2. Herzton sowie ein raues Holosystolikum (linker Sternalrand, thorakale Fortleitung). Im EKG ist ein überdrehter Linkslagetyp mit Zeichen der linksventrikulären Hypertrophie in den Brustwandableitungen (Volumenbelastung des linken Ventrikels) typisch. In der Echokardiografie finden sich eine fehlende Trikuspidalklappe, ein kleiner rechter Ventrikel und immer ein obligater Rechts-Links-Shunt auf Vorhofebene. Daneben sollten beurteilt werden:

- Dimension des VSD

- Ausprägung einer Pulmonalstenose

- ventrikuloarterielle Konkordanz oder Diskonkordanz

- zusätzliche Anomalien des Aortenbogens

Management. Bei einer duktusabhängigen Lungenoder Systemperfusion wird eine $\mathrm{PGE}_{1}$-Infusion gestartet. Ein restriktives Vorhofseptum kann ggf. mittels Ballonatrioseptostomie behandelt werden. Bei balancierten Verhältnissen mit milder Pulmonalstenose und mäßiger Zyanose sind die Neugeborenen oft in klinisch stabilem Zustand und es müssen zunächst keine Interventionen erfolgen. Eine Kreislauftrennung kann nur im stufenweise realisierten Fontankonzept gelingen (s. analog Kapitel „Hypoplastisches Linksherzsyndrom“).

\section{Herzfehler mit duktusabhängiger Systemperfusion (Linksherzobstruktion)}

\section{Kritische Aortenstenose}

Definition und Epidemiologie. Mit einer Häufigkeit von 3-6\% ist eine Fehlbildung der Aortenklappe einer der häufigeren Herzfehler mit einer Knabenwendigkeit (4:1) [8]. In Kombination mit subvalvulärer oder supravalvulärer Stenose (sinutubulärer Übergang) findet sich eine valvuläre Aortenstenose in bis zu 10\% der AHF. In 75\% findet sich eine bikuspide Klappe mit exzentrischer Restöffnung, seltener sind die unikuspide oder die trikuspid angelegte Klappe. Bei der neonatalen kritischen Aortenstenose ist die Klappe häufig myxö- 
matös verdickt mit zentraler „Pin-hole“-Öffnung und oft zusätzlicher Hypoplasie von Anulus, Aortenwurzel und Aorta ascendens. Die Klappe selbst repräsentiert dabei nur eine (wenngleich wichtige) Komponente der Stenose; Übergangsformen zum hypoplastischen Linksherzsyndrom bestehen.

\section{Anatomie und Hämodynamik}

Merke: Eine hochgradige Stenose kann bereits bei Neugeborenen zu einer Dekompensation des linken Ventrikels führen.

Hier spielt das Verhältnis von Nachlasterhöhung für den linken Ventrikel und dessen koronarer Perfusion eine entscheidende Rolle. Zudem kann eine sog. Endokard-Fibroelastose des linken Ventrikels bestehen, die häufig mit einer schweren diastolischen und systolischen linksventrikulären Funktionsstörung einhergeht.

Merke: Bei der kritischen Aortenstenose erfolgt über die stenostische Aortenklappe keine ausreichende Perfusion des Körperkreislaufs; die Systemperfusion ist duktusabhängig.

Klinik und Diagnostik. Ein meist beidseits parasternal maximal lautes raues Holosystolikum mit Fortleitung in die Carotiden und evtl. Ejektionsklick sowie eine paradoxe Spaltung des 2. Herztons sind bereits hinweisend. Bei schwerster valvulärer Aortenstenose und linksventrikulärer Funktionseinschränkung nimmt das Systolikum an Lautstärke ab. Insbesondere nach Duktusverschluss ist das Neugeborene zunehmend tachykard, mit reduzierter Blutdruckamplitude und schwachen peripheren Pulsen, grauem Hautkolorit und Tachydyspnoe durch ein sich entwickelndes Lungenödem. Im EKG finden sich Zeichen der Linksherzhypertrophie, nicht selten in Verbindung mit Erregungsrückbildungsstörungen.

Echokardiografisch wird der morphologische Typ der Klappenstenose festgelegt und die effektive Klappenöffnungsfläche abgeschätzt, die oft nicht einwandfrei bestimmbar ist. Der Klappenring wird bestimmt und weitere Linksstenosen sowie mögliche assoziierte Herzfehler ausgeschlossen.

Merke: Der Ductus arteriosus wird bzgl. Offenheit und Shuntrichtung analysiert, auch die Flussrichtung über dem Aortenbogen (antegrad oder nur retrograd) ist ein funktionell entscheidender Parameter für das weitere Notfallmanagement.
Wie in Abb.6a dargestellt, kann echokardiografisch eine Flussbeschleunigung über der stenotischen Aortenklappe nachweisbar sein.

Merke: Bei der kritischen Aortenstenose lässt die dopplerechokardiografisch bestimmte Flussgeschwindigkeit über die Aortenklappe keine sichere Aussage über den Schweregrad der Stenosierung zu. Bei beginnender oder manifester linksventrikulärer Funktionseinschränkung reduziert sich der Gradient ebenso wie bei offenem Ductus arteriosus und relevantem Rechts-Links-Shunt aus dem rechten Ventrikel in die deszendierende Aorta.

Bei relevantem Rechts-Link-Shunt über den Ductus arteriosus muss der linke Ventrikel nur ca. das halbe Herzzeitvolumen pumpen, woraus eine Reduktion des Gradienten erfolgt.

Der linke Ventrikel ist hypertrophiert und oft bereits funktionseingeschränkt bis hin zu dessen Unvermögen, eine nennenswerte Menge Blut über die stenosierte Klappe auszuwerfen. Konsekutiv erfolgt eine linksventrikuläre Dilatation. Gelegentlich imponiert ein an eine dilatative Kardiomyopathie erinnernder linker Ventrikel, verbunden mit verdickter Aortenklappe, reduziertem antegraden Fluss und stattdessen teilweise retrogradem Fluss im transversalen Aortenbogen als Hinweis auf eine Duktusabhängigkeit. Sehr wichtig ist noch die Analyse des Vorhofseptums von subkostal. Oft findet sich ein beschleunigter turbulenter LinksRechts-Shunt als Hinweis auf linksatriale Druckerhöhung infolge erhöhten enddiastolischen linksventrikulären Drucks und/oder einer Mitralklappenhypoplasie und -stenose, die nicht selten anzutreffen ist.

Management. Therapeutisch muss bei Neugeborenen mit duktusabhängiger Systemperfusion mit Hilfe einer $\mathrm{PGE}_{1}$-Infusion der Ductus arteriosus offengehalten werden. Nicht selten ist eine Katecholaminunterstützung der linksventrikulären Funktion und gelegentlich eine Beatmung erforderlich. Nach möglichst weitgehender Rekompensation wird katheterinterventionell eine antegrade oder retrograde Ballonvalvuloplastie der stenotischen Aortenklappe (Abb.6b) durchgeführt, die in ca. $70 \%$ eine relevante Druckreduktion ohne erhebliche residuelle Aorteninsuffizienz bewirken kann. Die Langzeitergebnisse dieser Intervention sind ungünstiger als bei der kritischen Pulmonalstenose. Die Freiheit von Re-Intervention liegt nach 5 Jahren nur bei ca. $60 \%$. 


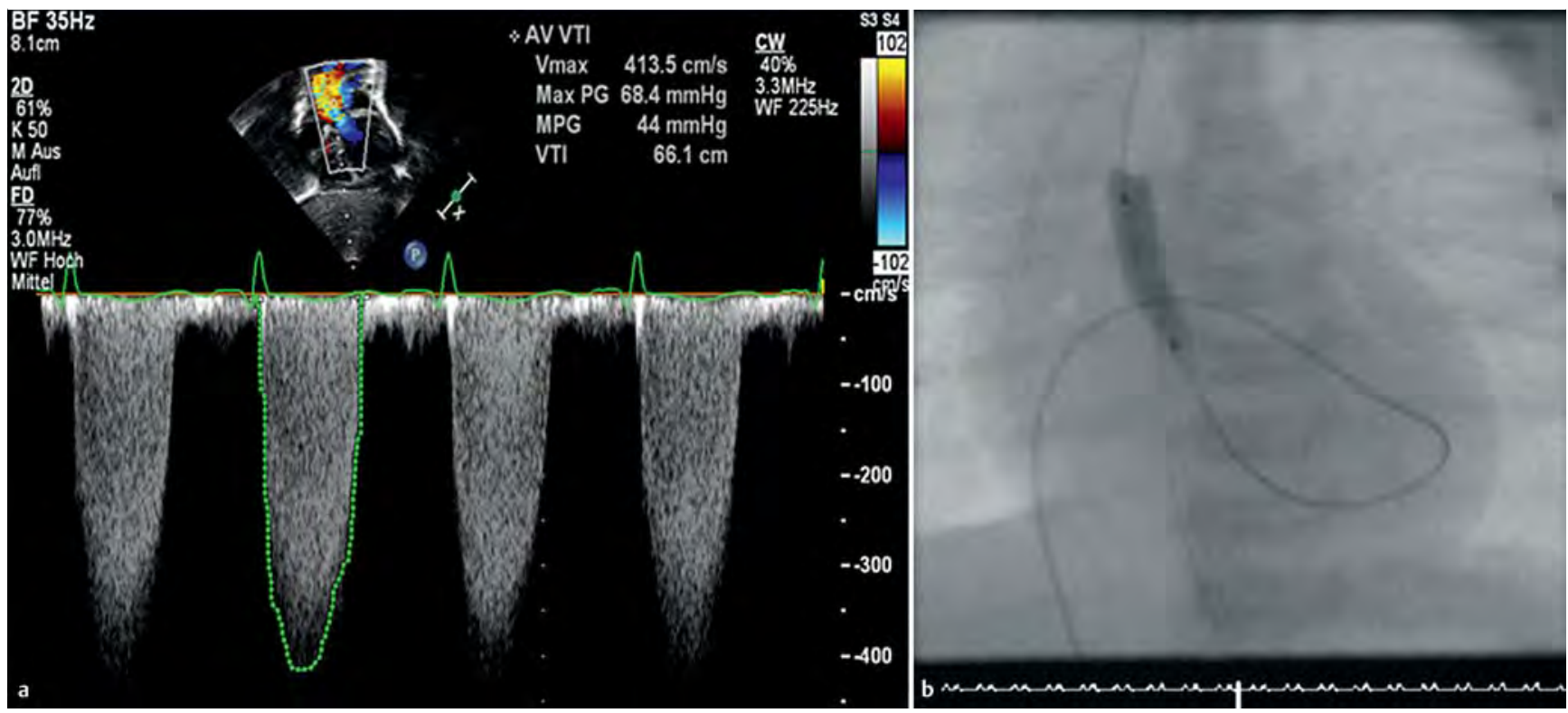

Abb. 6 Typischer echokardiografischer Befund (a) bei einem Neugeborenen mit kritischer Aortenklappenstenose und Durchführung einer Ballondilatation (b) der stenotischen Aortenklappe. a Echokardiografisch zeigt sich im 2D-Bild in der Einstellung von subxyphoidal das Bild einer hochgradigen Aortenklappenstenose. Im Farbdoppler wird eine Turbulenz distal der Klappe sichtbar, der CW-Doppler offenbart eine Flussbeschleunigung von 4,14 m/s. Die linke Herzkammer erscheint vergrößert mit eingeschränkter Funktion. Bei zunehmender linksventrikulärer Funktionseinschränkung nimmt die Flussbeschleunigung über die Aortenklappe ab. Daher lässt die echokardiografisch bestimmte Flussbeschleunigung keine Aussage über den Schweregrad einer kritischen Aortenklappenstenose zu. b Durch eine Ballondilatation kann eine signifikante Druckreduktion der Stenose erreicht werden, um eine Operation zu vermeiden oder den Zeitpunkt hinauszuzögern.

Bei frustraner Intervention oder primär zu hypoplastischem Klappenring oder bei zusätzlicher relevanter subaortaler Stenose kann bei geeigneter Koronaranatomie ein chirurgischer Ansatz mit Klappenersatz als Ross-Operation (bei der die Aortenklappe gegen die eigene Pulmonalklappe ausgetauscht und eine biologische Klappenprothese in Position der Pulmonalklappe implantiert wird), Ross-Konno-Operation (zusätzliche Beseitigung einer Subaortenstenose) oder als Homograft-Klappenersatz gewählt werden. Das Prozedurenrisiko dieser Eingriffe ist in gegenwärtigen kinderherzchirurgischen Datenbanken erheblich mit einer Mortalität von 15-25\% [9], in spezialisierten Einrichtungen jedoch deutlich geringer [10].

\section{Kritische Aortenisthmusstenose (ISTA)}

Definition und Epidemiologie. Eine Aortenisthmusstenose (ISTA) ist definiert als eine Einengung der Aorta im Übergangsbereich zwischen distalem Aortenbogen und Aorta descendens [11]. Eine solche Konstellation findet man bei etwa $7-10 \%$ der Kinder mit AHF und bei 0,2-0,6 von 1000 Lebendgeborenen. Es besteht eine Knabenwendigkeit (2:1). Dieses Krankheitsbild kann isoliert bestehen, ist jedoch nicht selten vergesellschaftet mit:

- Hypoplasie des Aortenbogens

- bikuspider Aortenklappe

- VSD
Ebenso zeigt sich eine Assoziation zu vielen anderen Herzfehlern.

Merke: Die intrauterine Diagnosestellung einer

ISTA ist oft schwierig (offener Ductus arteriosus!).

Anatomie und Hämodynamik. Nach der Lokalisation der aortalen Enge in Relation zur Mündung des Ductus arteriosus unterscheidet man eine präduktale, juxtaduktale und postduktale ISTA. Verschließt sich der Ductus arteriosus nicht direkt postnatal, präsentiert sich die ISTA oftmals erst im Alter von 2-4(-6) Wochen.

Bei frühzeitigem Duktusverschluss kann sich aufgrund der kritischen Druckbelastung des linken Ventrikels ein progredientes Linksherzversagen als Low-Cardiac-Output-Syndrom entwickeln. Typischerweise sind Nieren (akutes Nierenversagen), Leber (Gerinnungseinschränkung) sowie der Magen-Darm-Trakt (nekrotisierende Enterokolitis) aufgrund der Minderperfusion der unteren Körperhälfte und das zentrale Nervensystem (Gefahr der intrazerebralen Blutung) betroffen. Ein typischer Verlauf ist in der Kasuistik im ersten Teil in der Vorausgabe skizziert. 

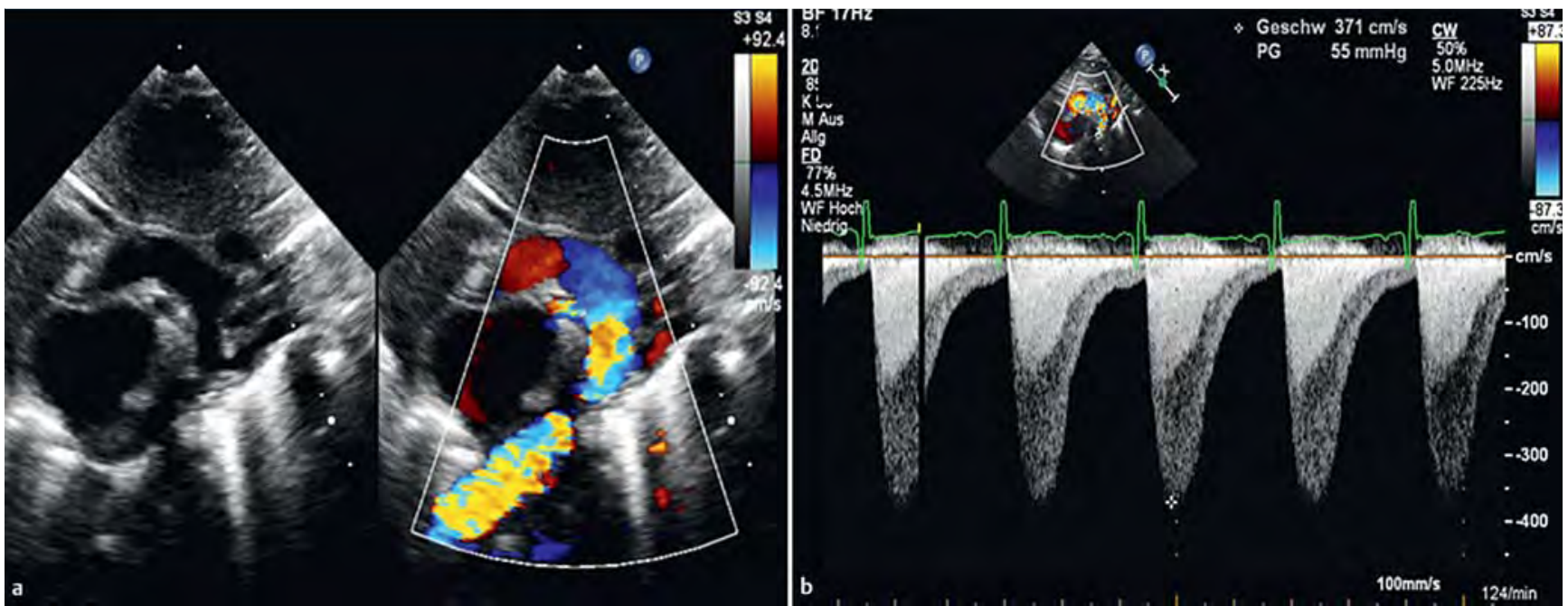

Abb. 7 Typische echokardiografische Befunde bei kritischer Aortenisthmusstenose. a Es zeigt sich im 2D-Bild von jugulär das Bild einer hochgradigen Einengung der deszendierenden Aorta etwas unterhalb des Abgangs der linken Arteria subclavia. b CW-Dopplerflusskurven durch die Stenose zeigen ein mit 3,7 m/s deutlich beschleunigten Fluss und ein charakteristisch ausgezogenes Flussprofil mit antegradem diastolischen Fluss („Sägezahnmuster“). Durch die sequenzielle Darstellung der Flüsse im gepulsten Doppler über den Verlauf vom distalen Aortenbogen bis zur Aortenisthmusstenose kann die Zunahme der Flussgeschwindigkeit von $1,8 \mathrm{~m} / \mathrm{s}$ auf $3,7 \mathrm{~m} / \mathrm{s}$ nachgewiesen werden.

Bei geöffnetem Ductus arteriosus kann dagegen der rechte Ventrikel die untere Körperhälfte versorgen. Es besteht dann ein systolischer Rechts-Links-Shunt mit konsekutiver Untersättigung der unteren Körperhälfte, die entsprechende Sauerstoffsättigungsdifferenz fällt im Pulsoxymetriescreening auf und sollte eine kinderkardiologische Untersuchung veranlassen.

Merke: Bei noch offenem Duktus können die Femoralispulse auch bei hochgradiger ISTA normal sein und ein Gradient fehlen. Allerdings ist dann die Sauerstoffsättigung der unteren Extremitäten im Vergleich zum rechten Arm erniedrigt (positives neonatales pulsoxymetrisches Screening!).

Klinik und Diagnostik. Die Säuglinge sind häufig bereits trinkschwach und zeigen typische Zeichen der frühkindlichen Herzinsuffizienz:

- vermehrtes Schwitzen

- Tachydyspnoe

- Tachykardie

- mangelnde Gewichtszunahme

In der Blutdruckmessung an allen 4 Extremitäten zeigt sich typischerweise ein relevanter Gradient ( $\geq 15-$ $20 \mathrm{mmHg}$ systolisch) mit erhöhten Blutdrücken an den Armen gegenüber den Beinen. Eine Diagnostik gelingt sehr zuverlässig mit der Echokardiografie (Abb.7), die in Zweifelsfällen durch eine CT-Angiografie oder - insbesondere bei Absicht der Notfallintervention - durch eine Herzkatheter-Angiografie zu ergänzen ist.
Chirurgisch wichtig sind insbesondere:

- Lokalisation und Morphologie der eigentlichen Isthmusstenose

- Größe und Hämodynamik des Ductus arteriosus (Shuntrichtung)

- Ausschluss weiterer begleitender Herzfehler

- korrekte Beschreibung des transversalen Aortenbogens (Lokalisation, Hypoplasie) und der supraaortalen Arterien

- Ausschluss einer Arteria lusoria (Fehlabgang der Art. subclavia dextra aus der Aorta descendens, anstelle aus dem Truncus brachiocephalicus; verläuft häufig dorsal der Speiseröhre)

Merke: Der Blutdruck des linken Armes kann bereits im Vergleich mit dem rechten Arm erniedrigt sein, wenn die linke Arteria subclavia im Bereich der Isthmusstenose entspringt und im Ursprungsbereich stenosiert ist. Eine weitere Variante ist möglich: Liegt eine postduktal abgehende Arteria lusoria vor, kann der Blutdruck des linken

Arms höher (prä-ISTA) als der des rechten Arms sein (post-ISTA).

Management. Beim Verdacht auf das Vorliegen einer ISTA sollte zunächst eine $\mathrm{PGE}_{1}$-Infusion begonnen werden. Weitere Maßnahmen, wie beispielsweise die Korrektur der metabolischen Azidose oder eine Applikation von positiv-inotropen Medikamenten bei eingeschränkter kardialer Funktion, sollten entsprechend der in der Vorausgabe beschriebenen Initialtherapie begonnen werden. 


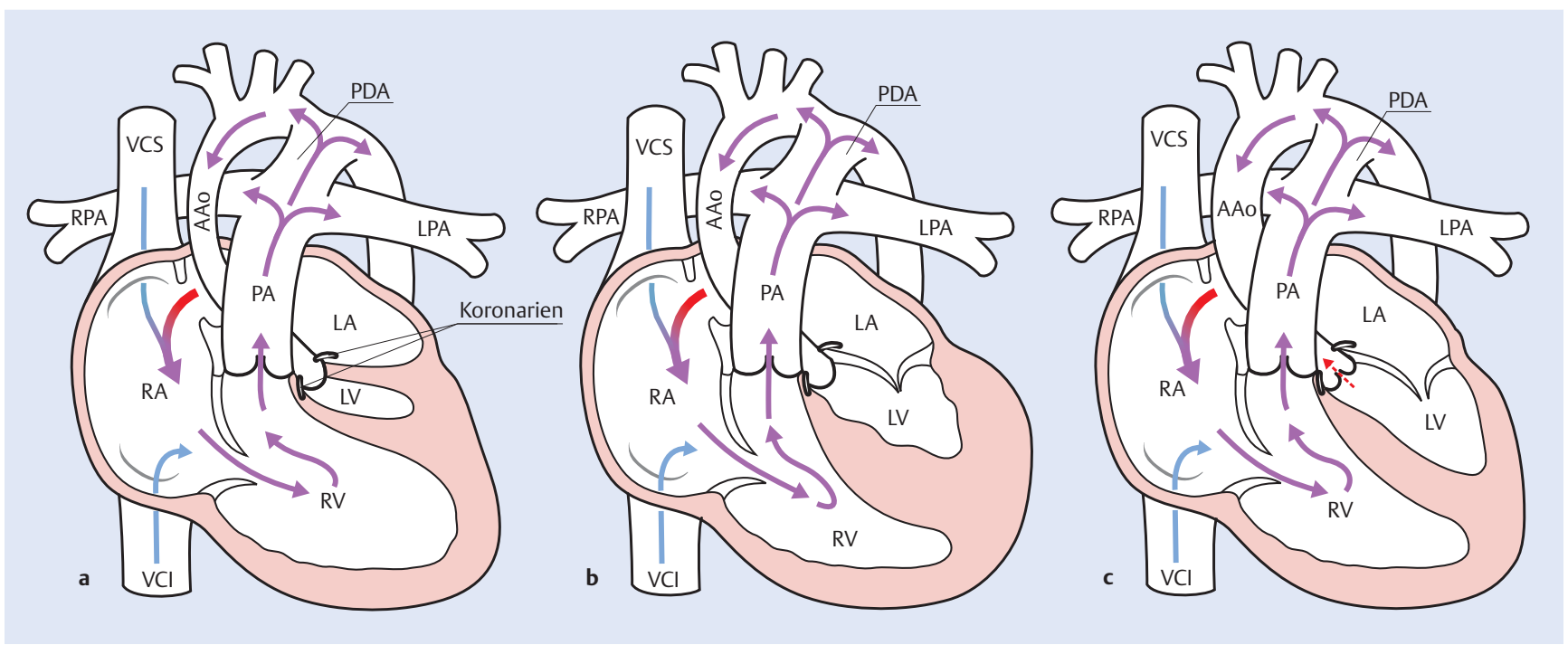

Abb. 8 Anatomische Varianten des hypoplastischen Linksherzsyndroms mit der Gemeinsamkeit des Unvermögens des hypoplastischen linken Ventrikels, ein ausreichendes Herzzeitvolumen für den Systemkreislauf zu generieren. a Mitralklappenatresie/Aortenklappenatresie. b Mitralklappenstenose/Aortenklappenatresie. c Mitralklappenstenose/Aortenklappenstenose. Das oxigenierte Blut aus den Lungenvenen erreicht über die Lücke im Vorhofseptum - ein ausreichend großer Defekt vorausgesetzt - den rechten Vorhof (roter Pfeil) und mischt sich hier mit dem desoxigenierten Blut der Systemvenen. Über den rechten Ventrikel werden sowohl die Lungenarterien wie auch über den offenen (!) Ductus arterious der Systemkreislauf mit dem geringer oxigenierten (violette Pfeile) Mischblut versorgt. Die ascendierende Aorta wie auch die Koronararterien werden retrograd über den Ducuts arteriosus perfundiert. In einigen Fällen ist ein geringer antegrader Fluss oxigenierten Blutes (gestrichelter roter Pfeil, Abb. c) über die stenostische Aortenklappe nachweisbar, der für die Koronarperfusion relevant sein kann; dieser kann aber niemals ein ausreichendes System-Herzzeitvolumen gewährleisten.

Merke: Die kritische ISTA mit Low-Cardiac-OutputSyndrom wird klinisch oft als „Sepsis“ fehlgedeutet. Eine sorgfältige klinische Untersuchung mit typischerweise fehlenden oder stark abgeschwächten Femoralispulsen könnte bereits die richtige Diagnose offenbaren.

Die weiteren Behandlungsoptionen der kritischen ISTA umfassen die zeitnahe chirurgische Korrektur. Bei typischer Lokalisation, ausreichend großem Aortenbogen sowie normaler Anatomie der Kopfhalsgefäße wird aktuell meist eine ISTA-Resektion mit anschließender angeschrägter „extended“ End-zu-End-Anastomose über eine linkslaterale Thorakotomie durchgeführt. Im Falle einer höhergradigen Aortenbogenhypoplasie kann aber auch eine Patchplastik des Aortenbogens über einen transsternalen Zugang mit Einsatz der HerzLungen-Maschine notwendig werden.

Merke: Bei Low-Cardiac-Output-Syndrom kann eine katheterinterventionelle Ballonangioplastie (ggf. mit Platzierung eines Stents) lebensrettend sein. Die definitive operative Versorgung erfolgt dann nach Rekompensation.

Nachteile der Ballonangioplastie sind neben möglichen Gefäßkomplikationen die höhere Rate an Rezidiven und die Entwicklung von Aortenaneurysmen im
Bereich der Dilatation. Auch bei optimaler Therapie verbleibt bei bis zu 30\% der Patienten eine persistierende arterielle Hypertonie.

\section{Hypoplastisches Linksherzsyndrom (HLHS)}

Definition und Epidemiologie. Unter dem Begriff „hypoplastisches Linksherzsyndrom“ sind Fehlbildungen des Herzens zusammengefasst, die mit einer Hypoplasie des linken Herzens (Mitralklappe, linker Ventrikel, Aortenklappe, aszendierende Aorta) einhergehen, die keine biventrikuläre Korrektur unter vernünftigen Risiko-Nutzen-Erwägungen zulassen (Abb. 8) [12].

Merke: Kennzeichnend für ein HLHS ist eine Duktusabhängigkeit der Systemperfusion sowie im Unterschied zur kritischen ISTA oder kritischen Aortenstenose die Unmöglichkeit, einen biventrikulären Kreislauf unter Nutzung der linken Herzseite chirurgisch oder interventionell zu etablieren.

Mit einer Häufigkeit von 1-2\% aller angeborenen Herzfehler ist es bei einer Inzidenz von 0,8-1\% für Herzfehler insgesamt zwar selten, stellt aber mit 10-20\% einen nicht unerheblichen Anteil an kritischen Herzfehlern dar. Chromosomale Auffälligkeiten bestehen bei ca. 10\% der Patienten. Kardiale Begleitfehlbildungen sind nicht selten vorhanden (z.B. Ventrikel- 


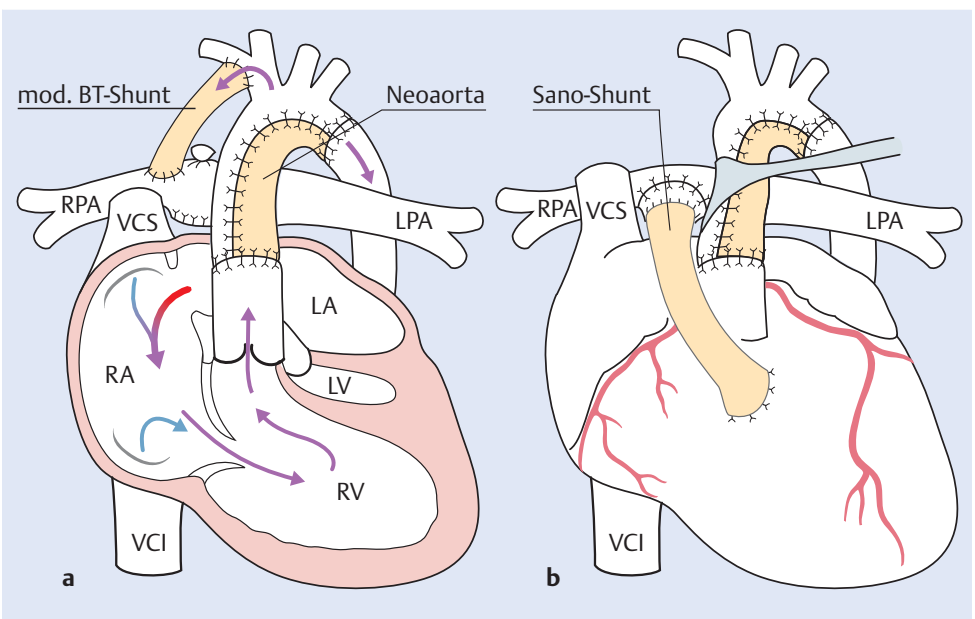

Abb.9 Norwood-Operation zur primären Palliation bei HLHS. a Norwood-OP mit modifiziertem Blalock-Taussig-(BT-)-Shunt. Die hypoplastische Aorta ascendens wird mit dem Pulmonalarterienhauptstamm anastomosiert (Seit-zu-Seit) und dieser distal unmittelbar vor der Pulmonalarterien-Bifurkation abgesetzt. Diese „Neoaorta“ wird im Bereich des distalen Aortenbogens zusätzlich plastisch erweitert. Über einen modifizierten BT-Shunt (Gefäßprothese von 3.5-4.0 mm Durchmesser zwischen A. subclavia rechts und rechter Pulmonalarterie) wird die Lungenperfusion (violetter Pfeil) sichergestellt. Nach Resektion des Vorhofseptums (wichtig: großzügige Atrioseptektomie!) kann das volloxigenierte pulmonalvenöse Blut (roter Pfeil) stauungsfrei zur rechten Herzseite abfließen, wo es sich mit dem desoxigenierten systemvenösen Blut (blaue Pfeile) vermischt. Damit wird der Systemkreislauf über die „Neoaorta“ mit geringer oxigeniertem Mischblut (Sauerstoffsättigung ideal um $80-85 \%$ ) (violette Pfeile) versorgt. b Norwood-OP mit Sano-Shunt (ventrikulopulmonaler Shunt).

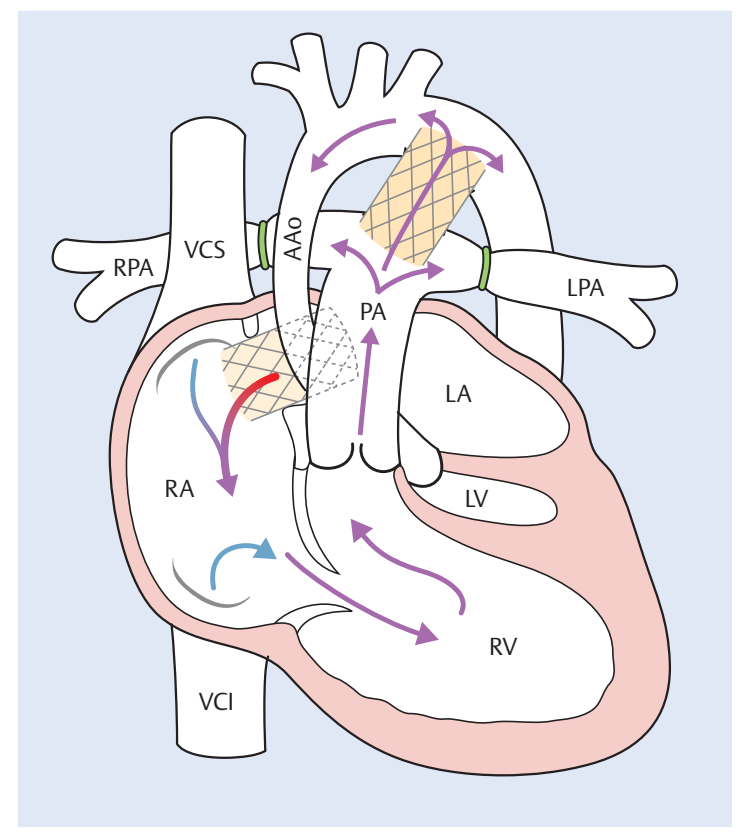

Abb. 10 Hybridverfahren zur primären Palliation bei HLHS als alternative Therapiemethode zur Norwood-OP. Das oxigenierte Blut aus den Lungenvenen erreicht über eine Lücke im Vorhofseptum - ein ausreichend großer Defekt vorausgesetzt - den rechten Vorhof (roter Pfeil). Optional kann zur Sicherstellung einer freien, nicht restriktiven Kommunikation zwischen den Vorhöfen - wie in der Abbildung gezeigt - ein Stent ins Vorhofseptum implantiert werden. Im rechten Vorhof mischt sich das oxigenierte Blut der Lungenvenen mit dem desoxigenierten Blut der Systemvenen (blaue Pfeile). Anschließend werden über den rechten Ventrikel sowohl die Lungenarterien wie auch über einen Stent im Ductus arterious der Systemkreislauf mit dem geringer oxigenierten Mischblut (violette Pfeile) versorgt. Ein bilaterales Banding der Pulmonalarterien (grün) verhindert eine pulmonale Überdurchblutung und ermöglicht eine Balance zwischen systemischer und pulmonaler Zirkulation. Die ascendierende Aorta wie auch die Koronararterien werden retrograd über den gestenten Ducuts arteriosus perfundiert. Bei Entwicklung einer Stenose im Aortenisthmus muss ggf. auch hier ein Stent implantiert werden (nicht in der Abbildung dargestellt), um eine Minderperfusion der Koronarien und Kopf-Hals-Gefäße zu verhindern.

linksventrikulären Unvermögens, ausreichend Blut in die Aorta auszuwerfen (z. B. linksventrikuläre Hypoplasie mit Endomyokard-Fibroelastose), nicht in der Lage, ein ausreichendes Herzzeitvolumen für den Systemkreislauf zu generieren. Die systemische Durchblutung erfolgt via Rechts-Links-Shunt aus dem rechten Ventrikel über den Ductus arteriosus (Abb.8). Das oxygenierte Blut, das sich aus den Lungenvenen in den linken Vorhof entleert, muss über das Vorhofseptum zum rechten Vorhof abfließen (Abb. 8, roter Pfeil).

Merke: Ein ausreichender Blutfluss sowohl im Bereich des Ductus arteriosus (Rechts-Links-Shunt) wie auch auf Höhe des Vorhofseptums (LinksRechts-Shunt) ist essentiell für das Überleben der betroffenen Neugeborenen.
Klinik und Diagnostik. Klinisch fallen die Neugeborenen mit den Zeichen eines progredienten kardiogenen Schockgeschehens auf, wobei initial eine Tachypnoe einziges Symptom sein kann. Die pulsoxymetrisch gemessene Sättigung ist oft aufgrund pulmonaler Hyperzirkulation über $90 \%$. Bleibt das Geschehen unbemerkt, kann das resultierende kardiogene Schockgeschehen zum raschen Tod des Kindes führen. Anzeichen dafür sind:

- blasses Hautkolorit

- arterielle Hypotension

- Oligurie

- metabolische Azidose

- nekrotisierende Enterokolitis 


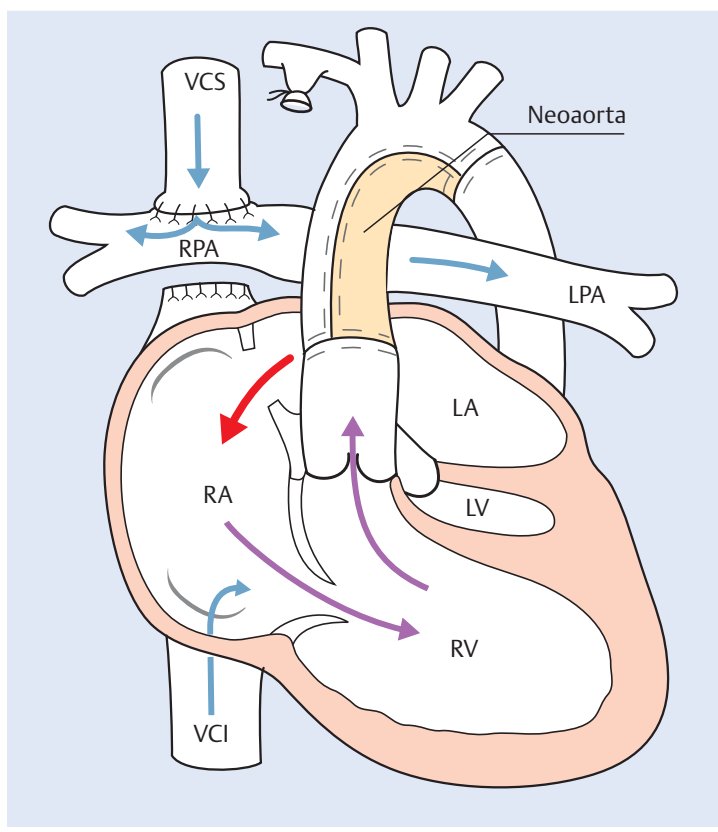

Abb. 11 Obere cavopulmonale Anastomose. Direkte Verbindung der oberen Hohlvene mit den Lungenarterien und Entfernung des systemisch-pulmonalen Shunts (modifizierter BT- oder SanoShunt, s. Abb.9). Das systemvenöse Blut der oberen Hohlvene (blaue Pfeile) fließt danach passiv durch die Lungen und mischt sich nach Oxigenierung (roter Pfeil) mit dem systemvenösen Blut aus der unteren Hohlvene (blauer Pfeil) im rechten Vorhof. Der Systemkreislauf erhält über die Neoarta weiterhin geringer oxigeniertes Mischblut, wobei die resultierende systemische Sauersoffsättigung zunächst unverändert bei etwa 80 - $85 \%$ liegen sollte (violette Pfeile). Hauptbenefit dieses Eingriff ist vielmehr die Wegnahme der Shunt-Rezirkulation und dadurch die wirksame Volumenentlastung des singulären rechten Ventrikels.

Management. Die Initialbehandlung basiert auf den in der Vorausgabe beschriebenen Therapieprinzipien einer duktusabhängigen Systemperfusion.

Merke: Da die Perfusion des gesamten Körpers über den Ductus arteriosus erfolgt, führt dessen Verschluss zu einer kritischen Minderperfusion des Körperkreislaufs bis hin zum kardiogenen Schock und konsekutiven Multiorganversagen.

Der Ductus arteriosus wird daher mittels PGE $_{1}$-Infusion offengehalten. Entscheidend für die weitere Intensivtherapie bis zu einer palliativen oder definitiven Chirurgie ist die Balance zwischen systemischem und pulmonalem Blutfluss. Durch die physiologische Abnahme des Lungengefäßwiderstands in den ersten Tagen post partum entwickelt sich eine kritische Abnahme des systemischen Blutflusses. Therapeutisch ist es daher von essentieller Bedeutung, jegliche Maßnahmen (s. Tab. 8 der Vorausgabe), die zu einer Abnahme des Lungengefäßwiderstands führen können (z.B. Sauerstoff, Ventilation mit niedrigem PEEP, respiratorische Alka-

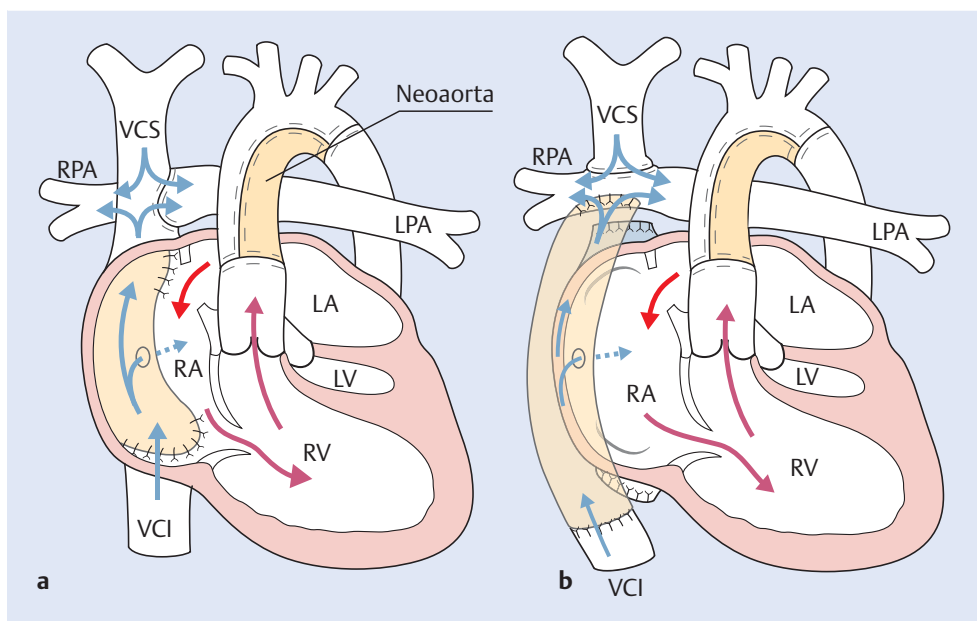

Abb. 12 Totale cavopulmonale Anastomose (TCPC, Fontanoperation). a TCPC mittels intrakardialen Patches. b TCPC mittels extrakardialen Tunnels. Bei dieser Operation als letztem Schritt der Norwood-Palliation werden der Lungen- und Körperkreislauf komplett voneinander getrennt. Auch das systemvenöse Blut der unteren Hohlvene (blaue Pfeile) wird nun direkt zu den Lungenarterien geleitet. Technisch wird dies entweder über einen extrakardialen (b) oder intrakardialen (a) Tunnel realisiert. Damit findet keine intrakardiale Mischung mehr statt und der Körperkreislauf wird entsprechend mit volloxigeniertem Blut versorgt. Einzige Ausnahme: Häufig wird der Fontan-Tunnel zum „pulmonalvenösen“ Vorhof fenestriert (Stanze von 5-6 mm), um in der perioperativen Phase der Kreislaufumstellung auf den komplett passiven Lungendurchfluss eine Überlaufmöglichkeit (gestrichelte blaue Pfeile) zum pulmonalvenösen Vorhof zu schaffen und dadurch kritische Reduktionen des System-Herzzeitvolumens zu vermeiden. Hieraus kann - je nach Nutzung des Überlaufs - eine leichte residuelle Zyanose resultieren (rotviolette Pfeile). Nach einigen Monaten verschließt sich die Fenestrierung meist spontan oder kann ggf. katheterinterventionell verschlossen werden.

lose), zu vermeiden, und evtl. Medikamente zur Senkung der systemischen Nachlast (z.B. Na-Nitroprussid) zu infundieren. Eine Sedierung z. B. mit Morphin 0,03$0,15 \mathrm{mg} / \mathrm{kg} / \mathrm{h}$ ist oft erforderlich, eine Pufferung mittels Bikarbonat sollte möglichst vermieden werden.

Die weiterführende Therapie am 3.-7. Lebenstag ist chirurgisch und besteht, wie in Abb. 9-12 gezeigt, aus einer mehrstufigen Kreislauftrennung nach dem Fontan-Prinzip.Alternativ kann eine Herztransplantation diskutiert werden. Eine anatomisch korrigierende Operation ist nicht möglich.

In jüngerer Zeit hat sich ein weiteres Konzept etabliert, das aus einem „Hybridansatz“ besteht und das vorwiegend Neugeborenen mit HLHS angeboten wird, bei denen eine Norwood-Operation als zu risikoreich angesehen wird [13]. Es besteht aus einem chirurgischen Banding (operative Einengung) beider Pulmonalarterien zur Kontrolle der pulmonalen Hyperperfusion in Verbindung mit einer katheterinterventionellen Implantation eines Duktusstents und ggf. einer Stentimplation in das Foramen ovale bei restriktivem Fluss über das Vorhofseptum (Abb.10). Bei fehlender antegrader Koronarperfusion muss ggf. der Aortenisthmus 
zusätzlich durch einen Stent offengehalten werden, um die retrograde Perfusion des Aortenbogens mit den Kopf-Hals-Arterien zu sichern und eine koronare Minderperfusion zu vermeiden. Diese Interventionen können zweizeitig durchgeführt werden: Zunächst ein bipulmonales Banding unter Belassen des $\mathrm{PGE}_{1}$, einige Tage später nach Erholung erfolgt dann die Stentimplatation in den Ductus arteriosus und ggf. in das Vorhofseptum im Herzkatheterlabor. Einige Gruppen bevorzugen ein einschrittiges Vorgehen, wobei der Chirurg nach dem bipulmonalen Banding eine Schleuse in den Pulmonalarterienhauptstamm einbringt, über die der Duktusstent antegrad implantiert wird.

Merke: Beim „Hybridansatz“ (Banding beider Pulmonalarterien, Duktusstent und ggf. Stentimplantation in das Vorhofseptum) wird die parallele „fetale“ Zirkulation gesichert und das Prostaglandin $E_{1}$ kann abgesetzt werden.

Sobald nach der Hybridintervention ein stabiler, balancierter, paralleler System- und Lungenkreislauf etabliert ist, können die Kinder für einige Zeit entlassen werden, bevor im Alter von 4-6 Monaten unter wesentlich günstigeren als den neonatalen Verhältnissen die sog. „Comprehensive-Stage-II“-Operation erfolgt:

- obere cavopulmonale Anastomose nach dem

Debanding (Abb. 11)

- Entfernung des Duktus- und Vorhofseptumstents

- Exzision des Vorhofseptums

- Rekonstruktion des Aortenbogens
Im weiteren Verlauf erfolgt die totale cavopulmonale Anastomose (Fontan-Operation) (Abb. 12) analog zum konventionellen chirurgisch gestuften Schema.

Die Lebenserwartung der Kinder mit HLHS nach dreischrittigem Fontankonzept ist mit einem 10-JahresÜberleben von etwa 60-70\% eingeschränkt, wobei allerdings Langzeitdaten aus dem Hybridkonzept noch fehlen. Aus diesem Grund sollte ein palliatives Therapiekonzept nach Diagnosestellung im Elterngespräch angesprochen werden, wird jedoch erfahrungsgemäß fast niemals gewählt. Die globale neurokognitive Entwicklung ist nicht signifikant schlechter als bei vergleichbaren Kindern mit biventrikulären Korrekturoperationen $[14,15]$.

\section{Herzfehler ohne nominelle Duktusabhängigkeit}

\section{Totale Lungenvenenfehlmündung (TAPVR)}

Definition und Epidemiologie. Die TAPVR findet man etwa bei 0,08/1000 Lebendgeborenen [1]. Oftmals wird die TAPVR in der pränatalen Echokardiografie nicht erkannt.

Anatomie und Hämodynamik. Man unterscheidet entsprechend der Lage der Mündung der fehlgeleiteten Lungenvenen 3 verschiedene anatomische Typen der TAPVR (Abb. 13).

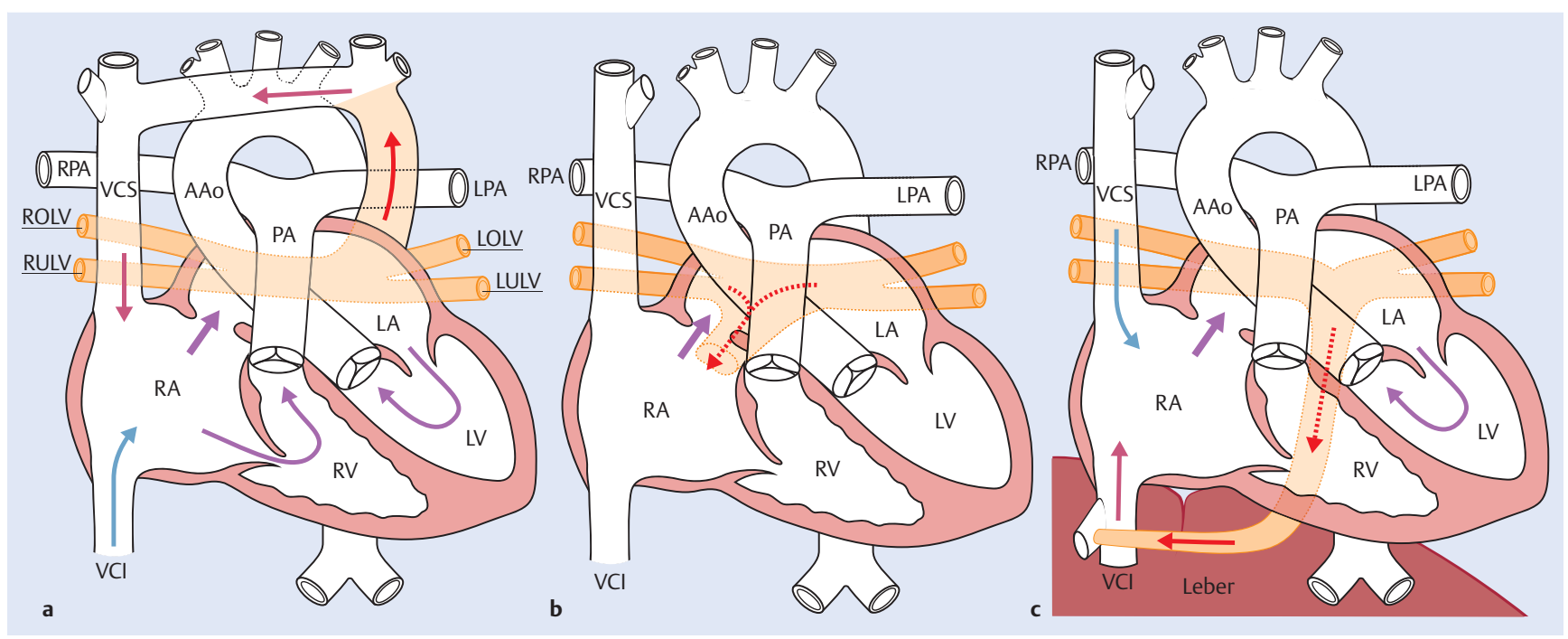

Abb. 13 Unterschiedliche anatomische Typen der TAPVR. Über das Vorhofseptum ist ein Rechts-Links-Shunt (violetter Pfeil) obligat. a Suprakardialer Typ: Drainage des Lungenvenenbluts (roter Pfeil) über die suprakardialen Systemvenen in den rechten Vorhof. b Kardialer Typ: Drainage des Lungenvenenbluts in den Koronarsinus und dessen Verlauf folgend in den rechten Vorhof. c Infrakardialer Typ: Drainage über ein nach kaudal verlaufendes Sammelgefäß mit Mündung in einen Pfortaderast oder über die Lebervenen via unterer Hohlvene in den rechten Vorhof. 
Die häufigste Form ist der suprakardiale Typ mit Drainage des Lungenvenenbluts zumeist über eine von vertikal nach kranial verlaufende Sammelvene mit Mündung über die Vena anonyma in die obere Hohlvene (Abb.13a und 15). Weniger häufig tritt der kardiale Typ mit Mündung der Sammelvene in den Koronarsinus oder (seltener) direkt in den rechten Vorhof auf (Abb.13b). Besonders schwierig gestaltet sich die Diagnostik des infrakardialen Typs mit Mündung der Sammelvene in die Leber in einen Pfortaderast und Drainage des Blutes über die Lebervenen und/oder den noch offenen Ductus venosus (Abb.13c). In seltenen Fällen findet sich kein Sammelgefäß, was zumeist mit einer Inoperabilität und einer schlechten Prognose einhergeht. In diesen Fällen kann das Lungenvenenblut über kleinste Kollateralen in umliegende Systemvenen drainieren. Es imponiert in diesem Fall zumeist eine schwere therapierefraktäre pulmonalvenöse Stauung und Hypoxämie, sodass die Kinder oft frühzeitig versterben.

Zusätzlich kann bei den verschiedenen Typen der TAPVR eine Behinderung des pulmonalvenösen Abflusses im Bereich der Pulmonalvenen, des Pulmonalvenensinus oder der Sammelvene vorliegen.

Merke: Die Ausprägung der Obstruktion des pulmonalvenösen Abstroms bestimmt die Klinik bei TAPVR.

Darüber hinaus sind Neugeborene mit TAPVR auf einen nicht restriktiven Blutfluss vom rechten in den linken Vorhof über das Vorhofseptum (Abb. 15d) angewiesen.
Klinik und Diagnostik. Entscheidend für die Schwere und Dynamik des klinischen Geschehens bei TAPVR ist das Ausmaß der Obstruktion des pulmonalvenösen Abstroms. Vereinfacht ergeben sich damit 2 unterschiedliche Verlaufsformen:

- TAPVR ohne Obstruktion: Ist der Abstrom des pulmonalvenösen Blutes nicht obstruiert, so kann sich das klinische Bild mit Verzögerung manifestieren und entspricht aufgrund der pulmonalen Rezirkulation dem klassischen Bild einer schweren shuntbedingten Herzinsuffizienz. Eine Zyanose ist nur gering ausgeprägt. Die Kinder fallen innerhalb der ersten Lebenswochen mit Trinkschwäche, Gedeihstörung, Schwitzen, Tachypnoe oder pulmonalen Infekten auf. Eine typische Verlaufsform ist in der Infobox „Kasuistik“ dargestellt.

- TAPVR mit Obstruktion: Aufgrund der nahezu immer bestehenden Unterversorgung des Systemkreislaufs und der respiratorischen Insuffizienz imponieren die betroffenen Neugeborenen oft sehr krank. Nach Geburt präsentieren sich eine zumeist schnell zunehmende Tachypnoe, metabolische Azidose und Kreislaufzentralisierung, zusammen mit einer therapierefraktären Zyanose. Die klinischen Symptome (Zyanose, Tachydyspnoe, Kreislaufinsuffizienz) können mit einer persistierenden pulmonalen Hypertonie des Neugeborenen (PPHN) verwechselt werden. Bei einer TAPVR mit relevanter Obstruktion des Pulmonalvenenabstroms kommt es zu einem rasch progredienten Lungenödem, oftmals mit den klinischen Zeichen eines schweren Lungenversagens. Das Röntgenbild zeigt eine massive Transparenzminderung (Abb.14a), das als Lungenpathologie fehlgedeutet

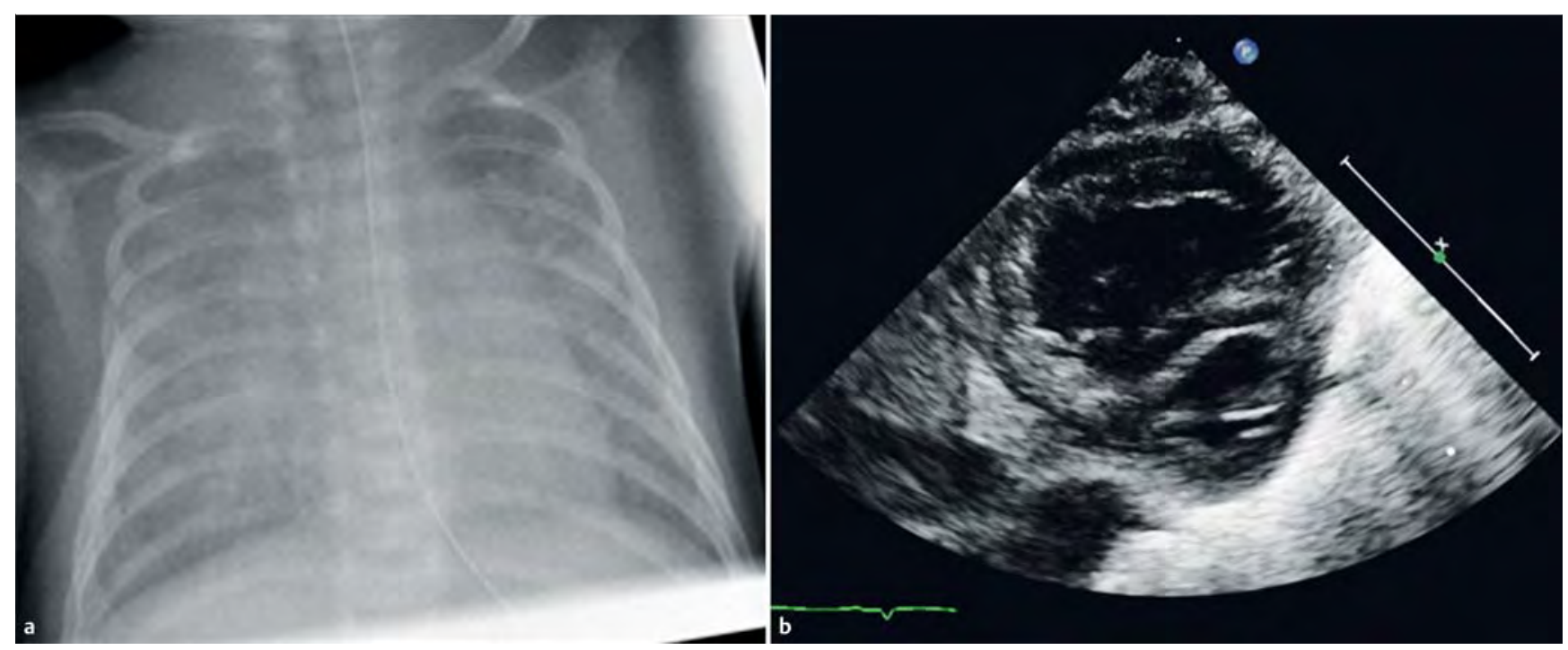

Abb. 14 Dekompensiertes Neugeborenes mit TAPVR vom infrakardialen Typ und Obstruktion des Lungenvenenabstroms. a Typisches Röntgenbild des Thorax. Es zeigte sich am 2. Lebenstag bei pulmonaler Rezirkulation und Obstruktion des Lungenvenenabstroms ein ausgeprägtes stauungsbedingtes Lungenödem, das bei vielen Patienten als primäre Lungenpathologie fehlgedeutet wird. b Echokardiografische Darstellung des rechten und linken Ventrikels in der parasternal kurzen Achse. Es zeigt sich aufgrund der mangelnden Füllung ein kleiner linker Ventrikel und als Zeichen der stark erhöhten rechtsventrikulären Volumen- und später auch Druckbelastung (s. abgeflachtes interventrikuläres Septum) ein großer, muskelstarker, rechter Ventrikel. 


\section{Kasuistik}

Der mittlerweile 8 Wochen alte Säugling wurde nach Überweisung durch den Kinderarzt aufgrund einer persistierenden Tachypnoe, Schwitzen und mangelndem Gedeihen beim niedergelassenen Kinderkardiologen vorgestellt. Aufgrund dieser Symptomatik erfolgte bereits zuvor eine kurzzeitige stationäre Aufnahme im Alter von 4 Wochen, bei der ein Infekt der oberen Luftwege als Ursache angenommen wurde. Bei der kinderkardiologischen Vorstellung bestand eine erhebliche Tachypnoe von 90-110/min, eine mit 4-5 Sekunden deutlich verlängerte Rekapillarisierungszeit sowie auskultatorisch ein deutlich betonter 2. Herzton und ein 2/6Systolikum mit Punctum maximum im 2./ 3. ICR parasternal links. Im EKG zeigte sich ein überdrehter Rechtstyp. Echokardiografisch stellte sich eine deutlich vergrö-
Berte und muskelstarke rechte Herzkammer mit erhöhtem, suprasystemischem Druck, abschätzbar über eine etwa zweitgradige Trikuspidalklappeninsuffizienz, dar. Es konnten bei sehr unruhigem Kind keine Lungenvenen dargestellt werden, die in den linken Vorhof mündeten. Auffällig war ein mit über $2 \mathrm{~m} / \mathrm{s}$ deutlich beschleunigter Fluss in der oberen Hohlvene (VCS). Aufgrund der Klinik überwies die Kollegin das Kind sofort in ein kinderkardiologisches Zentrum. Hier wurde eine totale Lungenvenenfehlmündung vom suprakardialen Typ diagnostiziert. Echokardiografisch ließen sich alle fehlmündenden Lungenvenen darstellen, ebenso der Sammelsinus und der Abstrom des Blutes über eine Vertikalvene und die Vena anonyma in die VCS (Abb. 15 b, c). Der Vorhofseptumdefekt war nicht restriktiv und es zeigte sich der obligate kräftige Rechts-Links-Shunt (Abb. 15d). Zur genauen Operationsplanung erfolgte zusätzlich eine Angiografie (Abb. 15a), die die echokardiografischen Befunde bestätigte. Am 2. Tag nach der Aufnahme erfolgte die Korrekturoperation mit Anschluss des Sammelsinus an den linken Vorhof. Der linke Ventrikel wurde durch ein temporäres Pacing über einen externen Herzschrittmacher und eine inotrop wirksame Therapie unterstützt. Am 1. Tag nach Operation konnte das Kind extubiert werden. Am 3. Tag erfolgte die Verlegung auf Normalstation. 14 Tage nach der Aufnahme konnte das Kind in gutem Allgemeinzustand nach Hause entlassen werden. werden kann. Aufgrund des verminderten Blutstroms zum linken Vorhof imponiert dieser ebenso wie der linke Ventrikel oftmals klein (Abb.14b). Der verringerte Zustrom zum linken Vorhof führt zu einer schweren Systemkreislaufinsuffizienz.

Merke: Klinik der TAPVR mit Obstruktion des Lungenvenenabstroms $=$ radiologische Lungenstauung + reduziertes Herzzeitvolumen.

In der Echokardiografie fällt in den typischen Schnittebenen die fehlende Konnektion der Pulmonalvenen an den linken Vorhof auf.

\begin{abstract}
Merke: Eine totale Lungenvenenfehlmündung wird selten pränatal diagnostiziert. Bei echokardiografischer Vergrößerung des rechten Ventrikels in Kombination mit einem kleinen linken Vorhof und einem Rechts-Links-Shunt auf Vorhofebene muss eine totale Lungenvenenfehlmündung gezielt ausgeschlossen werden.
\end{abstract}

In Abhängigkeit der Drainage des Lungenvenenflusses und des vorliegenden Typus der Fehlmündung (Abb.13) kann das Sammelgefäß zumeist eindeutig echokardiografisch identifiziert (Abb. 15) und der Verlauf dargestellt werden. Zur eindeutigen Darstellung der Lagebeziehung von Sammelvene zum linken Vorhof und damit zur Planung der Korrekturoperation ist neben der Echokardiografie (Abb.15b-d) oftmals eine Angio- grafie (Abb. 15a) im Herzkatheterlabor, eine kardiovaskuläre MRT oder CT sinnvoll.

\section{Management}

- TAPVR ohne Obstruktion: Bei Patienten ohne Obstruktion richtet sich das Vorgehen nach den klinischen Symptomen bei Diagnosestellung. Finden sich keine relevanten Gründe für ein abwartendes Verhalten wie Frühgeburtlichkeit, akute Infektionen oder führende Begleitfehlbildungen, so besteht aber auch hier aufgrund der Entwicklung einer pulmonalarteriellen Hypertonie die Indikation zur frühzeitigen Korrektur. Bis zur Operation ist häufig eine antikongestive Behandlung der Herzinsuffizienz nötig.

- TAPVR mit Obstruktion: Patienten mit pulmonalvenöser Obstruktion bedürfen aufgrund der zumeist sehr kritischen klinischen Situation (s.o.) eines schnellen und effektiven intensivmedizinischen Managements. Therapeutisch kann diesen Kindern kurzzeitig eine kontrollierte Beatmung mit hohem PEEP helfen, um dem Lungenödem entgegenzuwirken. Die Gabe von $\mathrm{PGE}_{1}$ in einem solchen Falle aggraviert durch Zunahme der Lungenperfusion die pulmonale Stauung und der klinische Zustand kann sich weiter verschlechtern.

Merke: Nach Diagnose einer TAPVR mit und ohne Obstruktion des Lungenvenenabstroms ist Prostaglandin $\mathrm{E}_{1}$ kontraindiziert. 


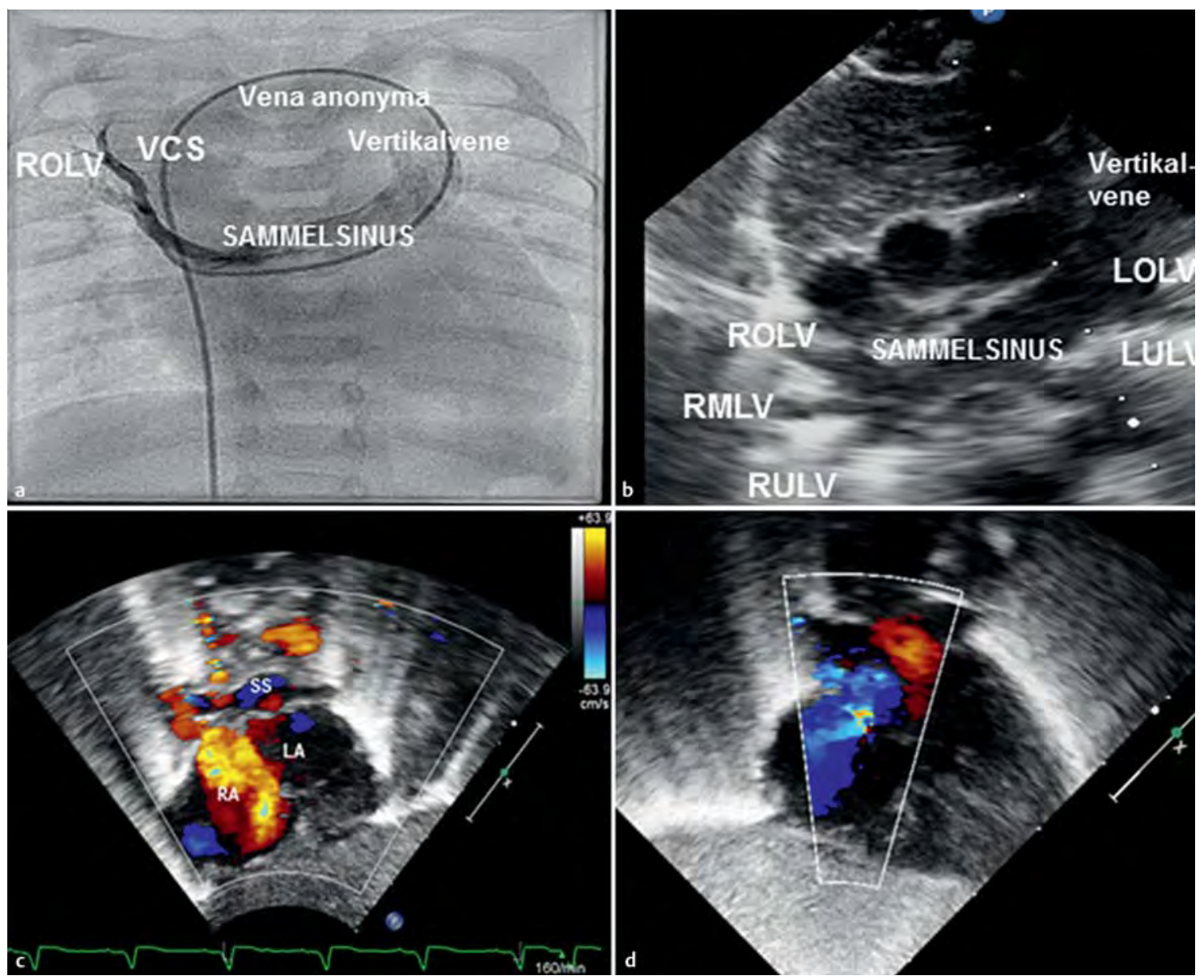

Abb. 15 Angiografische Darstellung und transthorakale Echokardiografie eines Patienten mit totaler Lungenvenenfehlmündung vom suprakardialen Typ ohne Obstruktion. a, b Angiografisch wie auch echokardiografisch lassen sich alle fehlmündenden Lungenvenen, die sich in einem Sammelsinus hinter dem linken Vorhof treffen, darstellen. Das Blut fließt von dort (dem eingebrachten Katheter folgend) in eine Vertikalvene und weiter über die Vena anonyma in die Vena cava superior (VCS) und schließlich in den rechten Vorhof. c Lagebeziehung zwischen linkem Vorhof und Sammelsinus von subkostal. d Obligater Rechts-Links-Shunt auf Vorhofebene.

Selten beruht die Systemkreislaufinsuffizienz auf einer Restriktion der Verbindung auf Vorhofebene. In diesem Fall kann durch ein Rashkind-Manöver die Kreislaufsituation temporär verbessert werden.

\footnotetext{
Merke: Eine TAPVR mit Obstruktion des Lungenvenenabstroms ist ein kinderkardiologischer/kinderkardiochirurgischer Notfall mit unmittelbarer Operationsindikation.
}

Ziel der operativen Therapie ist die Herstellung eines unrestriktiven Abstroms der fehlmündendenen Pulmonalvenen in den linken Vorhof. Dies erfolgt abhängig vom vorliegenden Typ (Abb. 13) zumeist durch den Anschluss des Sammelgefäßes an den linken Vorhof und den gleichzeitigen Verschluss des Vorhofseptumdefekts. Damit sind die Kreisläufe getrennt und die schwere Zyanose behoben. Die Prognose hängt im Wesentlichen neben der Erstversorgung von mög- lichen verbleibenden Stenosen im Lungenvenenabstrom oder im Bereich der neu geschaffenen Anastomose zum linken Vorhof ab. Liegen diese nicht vor und wurde die kritische Phase bis zur Diagnosestellung ohne sekundäre Organschäden überstanden, so ist die Prognose als günstig einzuschätzen.

\section{Transposition der großen Arterien (d-TGA)}

Definition und Epidemiologie. Mit einem Anteil von etwa $25 \%$ an den zyanotischen Herzfehlern des Neugeborenen ist die d-TGA nach der Fallot-Tetralogie der zweithäufigste zyanotische Herzfehler [16]. Neugeborene mit einer d-TGA sind zu zwei Drittel männlich und haben zumeist bei Geburt ein normales Gewicht. 
Anatomie und Hämodynamik. Durch den Fehlursprung der Aorta aus dem anatomisch rechten Ventrikel sowie der Pulmonalarterie aus dem anatomisch linken Ventrikel resultiert eine sog. ventrikuloarterielle Diskordanz (Abb. 16a-c). Der Terminus „d-TGA“ bezieht sich auf die Position der Aorta, die nach rechts ( $\mathrm{d}=$ dextro) und vorne verlagert ist und die Pulmonalarterie im Verlauf nicht kreuzt. Assoziierte kardiale Fehlbildungen sind in etwa 25\% der Fälle zu finden. Nur in Ausnahmefällen findet sich ein übergeordnetes Fehlbildungssyndrom (Tab. 1 der Vorausgabe). Als häufigste Begleitfehlbildungen findet man:

- VSD

- linksventrikuläre Ausflussbahnobstruktion

- ISTA

- mögliche Koronaranomalien

Merke: In ca. 75\% der Fälle besteht keine weitere kardiale Fehlbildung (sog. simple d-TGA).

Diese Bezeichnung wird auch verwendet, wenn Begleitfehlbildungen ohne eine hämodynamische Relevanz vorliegen, hierzu zählt auch ein kleiner, hämodynamisch unbedeutender VSD und ein persistierender Ductus arteriosus. Der Begriff „,komplexe“ $d$ TGA (25\% der Fälle) beschreibt das Vorliegen schwerwiegender assoziierter kardialer Fehlbildungen, u.a. einen großen VSD (ca. 20\%) oder eine relevante Ausflussbahnobstruktion (ca. 5\%).

Merke: Aufgrund der Parallelschaltung der Teilkreisläufe resultiert die Notwendigkeit von Kurzschlussverbindungen, um ein Mischen des oxygenierten Blutes mit dem venösen Blut zu gewährleisten.

Ein Überleben mit einer simple d-TGA (ohne VSD) ist demnach nur möglich, wenn die intrauterinen Kurzschlussverbindungen zwischen beiden Teilkreisläufen (Körper und Lunge) fortbestehen (Abb.16b): Foramen ovale und Ductus arteriosus. Postpartal muss ein ausreichend großer Defekt auf Vorhofebene (Abb.16b,d) obligat vorhanden sein, um dem sauerstoffreichen Blut aus dem linken Vorhof den Übertritt in den rechten Vorhof zu ermöglichen. Über einen offenen Ductus arteriosus mit Links-Rechts-Shunt nimmt der pulmonale Blutfluss zu (Abb.16b), demzufolge wird der Rückfluss über die Pulmonalvenen zum linken Vorhof erhöht und der Druck im linken Vorhof steigt, wodurch es zu einer verbesserten Mischung des sauerstoffreichen und -armen Blutes auf Vorhofebene kommt. Die Sättigung steigt in dem Maße, wie auf Vorhofebene ein Shunt vom linken zum rechten Vorhof möglich ist, wonach das sauerstoffreichere Blut über den rechten Ventrikel Zugang zur Aorta erhält.

Merke: Bei einer simple d-TGA mit Ductus arteriosus und nicht restriktivem Vorhofseptumdefekt füllt der Blutfluss über den Ductus arterious den linken Vorhof, woraus eine verbesserte Mischung des sauerstoffreichen mit dem sauerstoffarmen Blut auf Vorhofebene erfolgt. Demzufolge steigt die systemarterielle Sauerstoffsättigung.

Klinik und Diagnostik. Klinisch präsentiert das Neugeborene mit einer simple d-TGA neben einer generalisierten zentralen Zyanose häufig eine relevante Tachydyspnoe.

Merke: Ein zyanotisches Neugeborenes ohne Herzgeräusch und ohne pulmonale Ursache für die Zyanose hat bis zum Beweis des Gegenteils eine Transpositionsstellung der großen Arterien.

Die häufig im Verlauf zunehmende Zyanose ist in der Regel nicht oder nur minimal sauerstoffreagibel. Meist findet sich kein Herzgeräusch. Aufgrund der Anteposition des Systemventrikels ist häufig eine verstärkte präkordiale Pulsation zu tasten. Im Röntgen-Thorax finden sich eine Kardiomegalie und eine verstärkte Lungengefäßzeichnung. Es zeigt sich im Falle eines restriktiven Flusses über das Vorhofseptum oft auch eine schwere metabolische Azidose.

Cave: Besteht der Verdacht auf das Vorliegen einer d-TGA, liegt damit immer ein kinderkardiologischer Notfall vor, da der Sättigungsverlauf der weiteren Stunden nicht vorhersehbar ist und im Falle eines zunehmend restriktiveren Vorhofseptumdefekts nur mittels akuter interventioneller Maßnahmen suffizient behandelt werden kann.

In Abb. 17 sind die Einflussfaktoren auf das gute „Mischen“ auf Vorhofebene („good mixer“) wie auch Ursachen für das verringerte „Mischen“ („bad mixer“) mit ihren jeweiligen klinischen Folgen dargestellt.

Bei noch erhöhtem Lungengefäßwiderstand und dadurch bedingtem Fluss des arteriellen Blutes von der linken Kammer über die Pulmonalarterie und den offenen Ductus arteriosus (Rechts-Links-Shunt) in die deszendierende Aorta (Abb. 16b) stellen Neugeborene mit simple d-TGA die einzig denkbare Variante angeborener Herzfehler dar, bei denen es temporär zu einer höheren post- als präduktalen Sättigung kommen kann. Diese Kinder könnten im Rahmen des Puls- 


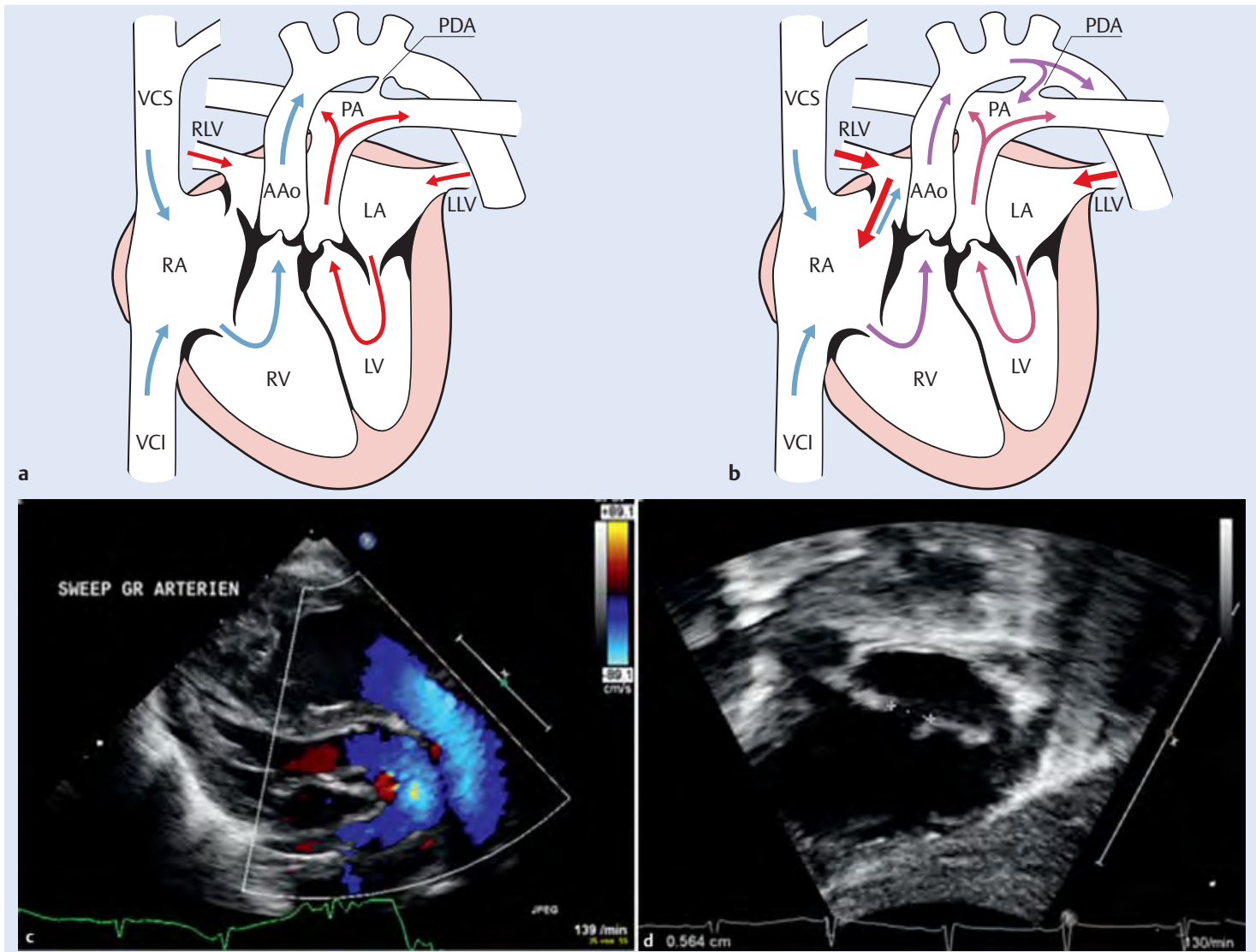

Abb. 16 Schematische Darstellung (a, b) und korrespondierende Echokardiografien (c, d) eines Neugeborenen mit simple d-Transposition der großen Gefäße. a In der schematischen Darstellung ist der Fehlursprung der Aorta aus dem anatomisch rechten Ventrikel sowie der Pulmonalarterie aus dem anatomisch linken Ventrikel, eine sog. ventrikuloarterielle Diskordanz, dargestellt. Bei verschlossenen fetalen Kurzschlussverbindungen (Foramen ovale, Ductus arteriosus) existiert eine Parallellschaltung der Kreisläufe, und eine Vermischung des desoxigenierten Blutes (blaue Pfeile) mit dem oxigenierten Blut (rote Pfeile) ist nicht möglich. Dieser Zustand ist nicht mit dem Leben vereinbar. b Die schematische Abbildung zeigt die Hämodynamik nach erfolgtem Rashkind-Manöver bei offenem Ductus arteriosus ( $\mathrm{PGE}_{1}$-Infusion!). Über den offenen Ductus arteriosus mit Links-Rechts-Shunt (violetter Pfeil) nimmt der pulmonale Blutfluss zu. In der Folge erhöht sich der Rückstrom des volloxigenierten Blutes (kräftige hellrote Pfeile) aus den Lungenvenen, wodurch es zu einer verbesserten Mischung auf Vorhofebene kommt. Durch die Zunahme des Links-Rechts-Shunts (kräftiger roter Pfeil) auf Vorhofebene (nicht restriktiver Vorhofseptumdefekt!) erhöht sich der Anteil des oxigenierten Blutes in der Aorta und die systemarterielle Sättigung (violette Pfeile) nimmt zu. c In der parasternal langen Achse sind die vorne liegende Aorta mit Ursprung aus dem rechten Ventrikel und die posterior liegende Pulmonalarterie mit der Bifurkation zu identifizieren. $\mathbf{d}$ Von subkostal stellt sich ein ausreichend großer Defekt zwischen beiden Vorhöfen nach Rashkind-Manöver dar.

oxymetriescreenings im Falle einer nur postduktalen Messung der Diagnosestellung entgehen.

Merke: Auch bei Neugeborenen mit einer d-TGA und einer begleitenden Aortenisthmusstenose kann die Perfusion der unteren Körperhälfte duktusabhängig sein und demzufolge an den unteren Extremitäten eine höhere Sättigung ableitbar sein.

Echokardiografisch lassen sich, wie in Abb. 16 gezeigt, die strukturellen Anomalien, evtl. Begleitfehlbildungen sowie die Shuntverbindungen zwischen Körperkreislauf und Lungenkreislauf (Größe des Ductus arteriosus bzw. Größe des Defekts im Vorhofseptum) darstellen. Die Echokardiografie ermöglicht in der Regel die Erhe- bung aller für die Planung der Korrekturoperation notwendigen Befunde. Ein diagnostischer Herzkatheter ist bei simple d-TGA deshalb nicht obligat, bei komplexen Begleitfehlbildungen zur Komplettierung der Darstellung der Anatomie z.B. der Koronararterien im Einzelfall aber weiterhin von Bedeutung.

Management. Als erste Maßnahme sollte eine $\mathrm{PGE}_{1^{-}}$ Infusion zum Wiedereröffnen bzw. Offenhalten des Ductus arteriosus begonnen werden. Bei einer schweren Zyanose sollte Sauerstoff appliziert werden. Dieser senkt zusätzlich den Lungengefäßwiderstand und führt zu einer Zunahme der Lungendurchblutung. Eine Intubation und leichte Hyperventilation können den Patienten ggf. weiter stabilisieren. Zur Vermeidung 


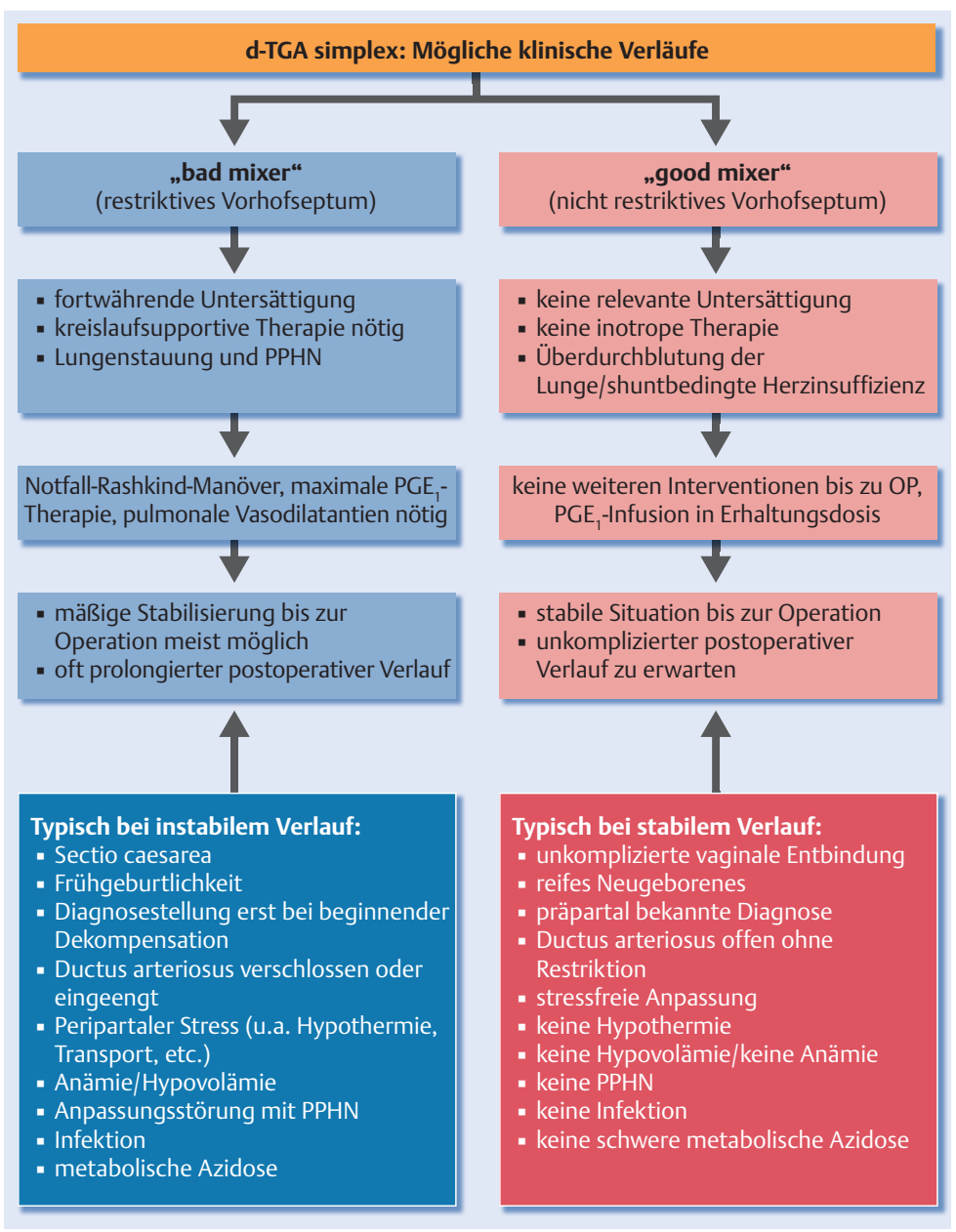

Abb. 17 Einflussfaktoren auf ein gutes („good mixer“) bzw. verringertes Mischen („bad mixer“) auf Vorhofebene bei einer simple d-TGA. Die Abbildung stellt Extremvarianten des klinischen Spektrums dar. PPHN = Persistierende pulmonale Hypertension des Neugeborenen. $\mathrm{PGE}_{1}=$ Prostaglandin $\mathrm{E}_{1}$.

einer kardialen Dekompensation bei einer simple d-TGA ist aber unabdingbar ein ausreichend weiter, nicht restriktiver Vorhofseptumdefekt für das Mischen auf Vorhofebene notwendig (Abb. 16 und 17). Dieser muss evtl. durch eine Ballonatrioseptostomie (Rashkind-Manöver) als Notfallmaßnahme geschaffen werden. Diese Prozedur kann ohne Angiografie unter echokardiografischer Kontrolle auf der Intensivstation über die Nabelvene durchgeführt werden. Alle Maßnahmen sind palliativ bis zur Operation.

Merke: Bei einer simple d-TGA mit restriktivem Vorhofseptumdefekt führt eine $\mathrm{PGE}_{1}$-Infusion zur klinischen Verschlechterung. Ein restriktiver Vorhofseptumdefekt stellt eine Indikation zum notfallmäßigen Rashkind-Manöver dar.
Bei der simple d-TGA erfolgt die Korrekturoperation durch Umsetzen der großen Arterien („arterial switch“) mit Transfer der Koronarien innerhalb der ersten 10 Lebenstage, wodurch eine anatomische Korrektur erreicht wird. Bei komplexer Transposition müssen evtl. zunächst Palliativoperationen bzw. Palliativkatheterinterventionen durchgeführt werden. Alternativ kommt als palliative Korrektur eine Vorhofumkehroperation in Frage (Umleitung der Blutströme auf Vorhofebene). Liegen keine begleitenden Fehlbildungen vor und konnte eine krisenhafte postpartale Verschlechterung mit möglichen negativen Spätfolgen im Sinne sekundärer Organschädigungen vermieden werden, so ist die Prognose als sehr gut zu bezeichnen.

\section{Kernaussagen}

- Die Mehrzahl der kritischen Herzfehler ohne begleitende Fehlbildungen zeigt in der Primärversorgung im Kreißsaal zunächst einen stabilen Zustand. Durch den postpartal noch erhöhten Gefäßwiderstand im kleinen Kreislauf, den initial noch offenen Ductus arteriosus und das offene Foramen ovale ist die Umstellung vom fetalen zum adulten Kreislauf noch nicht abgeschlossen. Behinderungen des pulmonalen oder systemischen Blutflusses können so zunächst noch kompensiert werden.

\section{- Bei kritischen Stenosevitien der Pulmonal- bzw.} Aortenklappe kann das Ausmaß der Flussbehinderung echokardiografisch unterschätzt werden, wenn die systolische Funktion des darunter liegenden Ventrikels bereits beeinträchtigt ist.

- Eine kritische Aortenisthmusstenose mit duktusabhängiger Perfusion der unteren Körperhälfte bedingt einen Rechts-Links-Shunt über den offenen Ductus arteriosus mit prä- und postduktaler Sättigungsdifferenz mit Untersättigung der unteren Körperhälfte.

Anders bei duktusabhängiger Lungenperfusion: Hier erfolgt über den Duktus arteriosus ein Links-RechtsShunt, entsprechend findet sich daher in dieser Konstellation keine prä- und postduktale Sättigungsdifferenz.

- Bei einer Fallot-Tetralogie bestimmt der Grad der Obstruktion von rechtsventrikulärem Ausflusstrakt und den Pulmonalstenosen das Ausmaß des intrakardialen Rechts-Links-Shunts und damit die Ausprägung der zentralen Zyanose. Bei nur geringer Obstruktion sind die Neugeborenen gering zyanotisch bis azyanotisch (sog. „Pink Fallot“). Innerhalb der ersten Lebensmonate entwickelt sich jedoch eine zunehmende Stenosierung mit Zunahme der Zyanose. 
- Bei einer d-Transposition der großen Arterien ohne Begleitvitien (z. B. Ventrikelseptumdefekt, Pulmonalstenose) muss postpartal ein ausreichend großer Defekt auf Vorhofebene vorhanden sein oder mittels interventioneller Maßnahmen (Rashkind-Manöver) geschaffen werden, um einen Kreuzshunt auf Vorhofebene und damit eine ausreichende Oxigenierung zu ermöglichen. Über einen Links-Rechts-Shunt durch den Ductus arteriosus wird zusätzlich das Angebot desoxigenierten Bluts zur Lunge verbessert und die Füllung des linken Vorhof gesteigert, wodurch eine verbesserte Mischung des sauerstoffreichen mit dem sauerstoffarmen Blut auf Vorhofebene über den Defekt erfolgt und damit die systemarterielle Sauerstoffsättigung steigt.

- Nach der Fallot-Tetralogie und der d-Transposition der großen Arterien ist die Trikuspidalatresie der dritthäufigste zyanotische Herzfehler. Bei diesem Herzfehler kann je nach Anatomie der ventrikuloarteriellen Verbindungen und der großen Arterien eine Duktusabhängigkeit für den Pulmonalkreislauf (Normalstellung der großen Arterien) oder den Systemkreislauf (Transpositionsstellung der großen Arterien) bestehen. Auch balancierte Kreisläufe ohne Duktusabhängigkeit sind möglich.

- Beim hypoplastischen Linksherzsyndrom ist der „Hybrid-Ansatz“ ein alternatives Therapiekonzept zur Norwood-Operation, insbesondere für Patienten mit zusätzlichen letalitätswirksamen Risikofaktoren in der Neonatalperiode. Durch chirurgisches Banding beider Pulmonalarterienäste wird eine Balancierung zwischen der Lungen- und Systemperfusion nach Abfall des Lungengefäßwiderstands angestrebt. Durch eine Implantation eines Duktusstents und ggf. nötige Beseitigung einer Restriktion auf Vorhofebene (Stent, chirurgisch) wird die fetale Zirkulation erhalten. Oft kann auf diese Weise die Palliation mittels einer Comprehensive-Stage-II-Operation (siehe Text) in das spätere Säuglingsalter verschoben werden.

- Neugeborene mit einer totalen Lungenvenenfehlmündung und Obstruktion des Pulmonalvenenabstroms sind mit konservativen Therapiemaßnahmen kaum zu stabilisieren. Eine Zyanose entsteht durch den obligaten Rechts-Links-Shunt auf Vorhofebene und das durch die resultierende Stauung hervorgerufene pulmonale Ventilations-Perfusions-Mismatch. Die pulmonalvenöse Obstruktion bedingt zusätzlich eine kritische Reduktion des Systemherzzeitvolumens. Eine Prostaglandin- $\mathrm{E}_{1}$-Infusion kann über eine Zunahme der Lungenperfusion und letztendlich der Lungenstauung die klinische Situation verschlechtern und ist daher kontraindiziert. Die Kombination aus einer schweren Zyanose mit Lungenstauung und Sys- temkreislaufinsuffizienz macht eine schnellstmögliche Operation unumgänglich. Bei primär sehr instabilen Patienten kann eine palliative Katheterintervention mit Stenten der pulmonalvenösen Obstruktion indiziert sein.

Interessenkonflikt: Prof. Beerbaum - keine Interessenkonflikte, Dr. Köditz - Vortragshonorare, Erstattung von Kongressgebühren und Reisekosten durch die Xenios AG, Deutschland, Dr. Jack und Dr. Böhne - Vortragshonorare, Übernahme von Kongressgebühren und Reisekosten durch die B. Braun Melsungen AG, Deutschland, und die Pall GmbH, Deutschland.

\section{Über die Autoren}

\section{Martin Böhne}

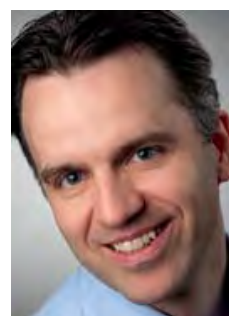

Jahrgang 1976, Dr. med. Nach dem Medizinstudium in Hannover Facharztausbildung an der Kinderklinik der Medizinischen Hochschule Hannover. Ausbildung in Kinderkardiologie und pädiatrischer Intensivmedizin. Funktionsoberarzt auf der interdisziplinären pädiatrischen Intensivstation der Abteilung für Pädiatrische Kardiologie und Intensivmedizin der $\mathrm{MHH}$.

\section{Thomas Jack}

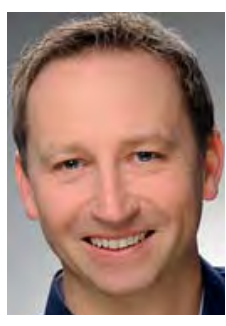

Jahrgang 1973, Dr. med. Nach dem Medizinstudium in Marburg und Hannover, Absolvierung der Facharztausbildung an der Kinderklinik der Medizinischen Hochschule Hannover. Seit 2012 tätig als Oberarzt der Abteilungpädiatrische Kardiologie und Intensivmedizin. Tätigkeitsbereich ist die interdisziplinäre pädiatrische Intensivstation der $\mathrm{MHH}$.

\section{Harald Köditz}

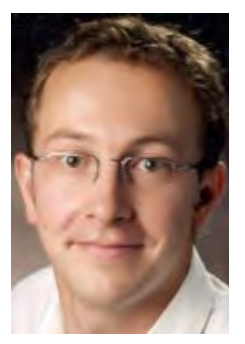

Jahrgang 1971, Dr.med. Nach dem Medizinstudium und der Facharztausbildung in Hannover seit 2003 als Oberarzt an der Kinderklinik der Medizinischen Hochschule Hannover tätig. Anstellung im Great Ormond Street Hospital for Children, London, UK 2004. Weiterbildungsermächtigung für spezielle pädiatrische Intensivmedizin. 


\section{Philipp Beerbaum}

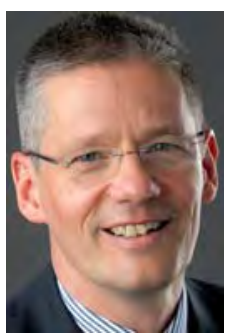

Jahrgang 1962, Univ.-Prof. Dr. med. Nach Medizinstudium, Promotion und Facharztzeit in Köln Ausbildung als Intensivmediziner und Kinderkardiologe im Herz- und Diabeteszentrum NRW (Bad Oeynhausen), dort Oberarzt und Habilitation an der Ruhr-Universität Bochum. Ab 2006 für 5 Jahre in London (UK) als Consultant Paediatric Cardiologist im Evelina Children's Hospital und Senior Lecturer in Paediatric Cardiovascular Sciences im Department of Imaging Sciences \& Biomedical Engineering am King's College London; anschließend Radboud Universiteit, Nijmegen. Seit 2012 Direktor der Klinik für Pädiatrische Kardiologie und Intensivmedizin an der Medizinischen Hochschule Hannover.

\section{Korrespondenzadresse}

Prof. Dr. med. Philipp Beerbaum

Medizinische Hochschule Hannover

Pädiatrische Kardiologie und Intensivmedizin

Carl-Neuberg-Str. 1

30625 Hannover

E-Mail: Beerbaum.Philipp@mh-hannover.de

\section{Literatur}

1 Da Cruz E, Ivy D, Jaggers J. Pediatric and Congenital Cardiology, Cardiac Surgery and Intensive Care. London: Springer; 2014

2 Jux C, Weil J, Dalla Pozza R, Bennink G, Horke A. Leitlinie Pädiatrische Kardiologie: Valvuläre Pulmonalstenose. Deutsche Gesellschaft für Pädiatrische Kardiologie; 2010 (http://www.kinderkardiologie.org/dgpkLeitlinien.shtml)

3 Weil J, Bertram H, Sachweh JS. Leitlinie Pädiatrische Kardiologie: Fallot'sche Tetralogie. Deutsche Gesellschaft für Pädiatrische Kardiologie; 2011 (http://www.kinderkardiologie.org/ dgpkLeitlinien.shtml)

4 Rudolph AM. Congenital Diseases of the Heart: Clinical-physiological Considerations. 3rd edn. New York: Wiley-Blackwell; 2009
5 Chubb H, Pesonen E, Sivasubramanian S et al. Long-term outcome following catheter valvotomy for pulmonary atresia with intact ventricular septum. J of the Am Coll of Card 2012; 59: $1468-1476$

6 Alwi M. Management algorithm in pulmonary atresia with intact ventricular septum. Catheterization and cardiovascular interventions. Offic J Soc for Card Angiogr \& Interv 2006; 67: $679-686$

7 Yoshimura N, Yamaguchi M. Surgical strategy for pulmonary atresia with intact ventricular septum: initial management and definitive surgery. Gen Thor and Card Surg 2009; 57 : $338-346$

8 Ewert P, Horke A, Haas NA. Leitlinie Pädiatrische Kardiologie: Aortenklappenstenose. Deutsche Gesellschaft für Pädiatrische Kardiologie; 2011 (http://www.kinderkardiologie.org/ dgpkLeitlinien.shtml)

9 Woods RK, Pasquali SK, Jacobs ML et al. Aortic valve replacement in neonates and infants: an analysis of the Society of Thoracic Surgeons Congenital Heart Surgery Database. J of Thor and Card Surg 2012; 144: 1084-1089

10 Aszyk P, Thiel C, Sinzobahamvya N et al. Ross-Konno procedure in infants: mid-term results. Eur ] of Card-Thor Surg: Off ] of the Eur Ass for Card-Thor Surg 2012; 42: 687-694

11 Haas NA, Ewert P, Hager A, Schlensak C. Leitlinie Pädiatrische Kardiologie: Aortenisthmusstenose. Deutsche Gesellschaft für Pädiatrische Kardiologie; 2011 (http://www.kinderkardiologie.org/dgpkLeitlinien.shtml)

12 Haas NA, Jux C, Photiadis J, Kramer HH. Leitlinie Pädiatrische Kardiologie: Hypoplastisches Linksherzsyndrom (HLHS). Deutsche Gesellschaft für Pädiatrische Kardiologie; 2013 (http://www.kinderkardiologie.org/dgpkLeitlinien.shtml)

13 Schranz D, Bauer A, Reich B et al. Fifteen-year Single Center Experience with the „Giessen Hybrid“ Approach for Hypoplastic Left Heart and Variants: Current Strategies and Outcomes. Pediatr Cardiol 2015; 36: 365-373

14 Gaynor JW, Ittenbach RF, Gerdes M et al. Neurodevelopmental outcomes in preschool survivors of the Fontan procedure. J of Thor and Card Surg 2014; 147: 1276-1282

15 Goldberg CS, Schwartz EM, Brunberg JA et al. Neurodevelopmental outcome of patients after the fontan operation: A comparison between children with hypoplastic left heart syndrome and other functional single ventricle lesions. J of Ped 2000; 137: 646-652

16 Rickers C, Horke A, Paul T. Leitlinie Pädiatrische Kardiologie: D-Transposition der großen Arterien. Deutsche Gesellschaft für Pädiatrische Kardiologie; 2013 (http://www.kinderkardiologie.org/dgpkLeitlinien.shtml) 


\section{CME-Fragen}

\section{CME.thieme.de}

CME-Teilnahme

- Viel Erfolg bei Ihrer CME-Teilnahme unter http://cme.thieme.de

- Diese Fortbildungseinheit ist 12 Monate online für eine CME-Teilnahme verfügbar.

- Sollten Sie Fragen zur Online-Teilnahme haben, unter

http://cme.thieme.de/hilfe finden Sie eine ausführliche Anleitung.

1

Welche diagnostische Maßnahme oder welcher Befund erbringt keine Hinweise auf das Vorhandensein einer kritischen Aortenisthmusstenose?
A Pulstasten an oberer und unterer Extremität
B oszillometrisch gemessener höherer Blutdruck der unteren Extremität
C Darstellung des Aortenisthmusbereichs mittels transthorakaler Echokardiografie
D Sättigungsdifferenz zwischen oberer und unterer Extremität
E pathologisches Flussprofil in der Bauchaorta oder den abdominellen Gefäßen

\section{2}

Welche Aussage trifft nicht zu? Zu den kritischen Herzfehlern des Neugeborenen kann gehören:
A Aortenisthmusstenose (ISTA)
B Transposition der großen Arterien (TGA)
C hypoplastisches Linksherzsyndrom (HLHS)
D persistierendes Ductus arteriosus (PDA)
E Pulmonalatresie (PA)

\section{3}

Welche Aussage zur interventionellen Therapie von angeborenen Herzfehlern ist nicht richtig?
A Die interventionelle Eröffnung des Vorhofseptums (Rashkind-Manöver) kann bei einer Transpositionsstellung der großen Gefäße (TGA) und kritischer Hypoxämie ein lebensrettender Eingriff sein.

B Die kritische valvuläre Aortenstenose kann mit einer Ballonvalvuloplastie behandelt werden.

C Der Ballonkatheter für die Erweiterung des Vorhofseptumdefekts bei der TGA kann durch die Nabelvene eingeführt werden.

D Bei einer kritischen Aortenisthmusstenose kann eine Gefäßstütze (Stent) in die Engstelle eingebracht werden.

E Das Rashkind-Manöver bei Kindern mit TGA und restriktivem Vorhofseptum kann nur im Herzkatheterlabor unter Durchleuchtung durchgeführt werden.
Bei welcher Pathologie findet sich echokardiografisch typischerweise kein Rechts-Links-Shunt über dem Vorhofseptum?
A persistierende pulmonale Hypertension des Neugeborenen

B totale Lungenvenenfehlmündung

C Hypoplastisches Linksherzsyndrom (HLHS)

D Trikuspidalatresie

E Pulmonalatresie mit intaktem Ventrikelseptum 


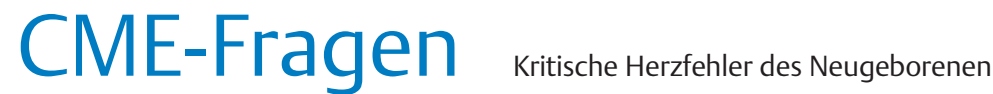

5

Welche Aussage trifft nicht zu?

A Bei Neugeborenen mit einer totalen Lungenvenenfehlmündung kann eine Infusion mit Prostaglandin $\mathrm{E}_{1}$ die klinische Symptomatik verschlechtern.

B In einigen Fällen kann man bei Neugeborenen mit einer kritischen Pulmonalstenose das raue, spindelförmige Systolikum mit Punctum maximum im 2./3. ICR links parasternal nicht auskultieren.

C Bei Neugeborenen mit einer Trikuspidalatresie findet man häufig einen überdrehten Linkstyp im 12-Kanal-EKG.

D Bei einem Neugeborenen mit einer kritischen Aortenisthmusstenose finden sich in der klinischen Untersuchung keine oder nur abgeschwächte Pulse in der Leiste sowie ein relevanter systolischer Blutdruckgradient ( $\geq 15-20 \mathrm{mmHg}$ ) mit erhöhten Blutdrücken an den Armen gegenüber den Beinen.

E Neugeborene mit einer kritischen Aortenstenose zeigen echokardiografisch häufig verdickte Aortenklappensegel und eine hyperdyname Funktion des linken Ventrikels.

6

Welche Aussage zu einer totalen Lungenvenenfehlmündung (TAPVR) ist nicht korrekt?
A Entsprechend der Mündung der fehlgeleiteten Lungenvenen unterscheidet man 3 verschiedene anatomische Typen der TAPVR.

B Die klinischen Symptome können denen einer persistierenden pulmonalen Hypertonie des Neugeborenen (PPHN) entsprechen und damit verwechselt werden.

C Bei einer TAPVR mit Obstruktion des Lungenvenenabstroms zeigt sich neben klinischen Zeichen der Systemkreislaufinsuffizienz im Röntgen-Thorax eine globale Zunahme der Transparenz in den Lungenfeldern.

D Bei einer TAPVR mit Obstruktion des Lungenvenenabstroms besteht eine Notfallindikation zur operativen Korrektur.

E Echokardiografisch finden sich ein kleiner linker Ventrikel, ein Rechts-Links-Shunt über das interatriale Septum sowie oftmals ein Sammelsinus in enger Lagebeziehung zum linken Vorhof.

7

Welche Aussage zu einem hypoxämischen Anfall bei einer Fallot-Tetralogie ist nicht richtig?
A Der hypoxämische Anfall kann durch körperliche oder psychische Belastung ausgelöst werden. Oftmals tritt er auch nach dem Füttern oder beim Baden auf.

B Bei Persistenz der Zyanose ist nach Versagen der initialen Behandlungsmaßnahmen eine intravenöse Applikation von Na-Nitroprussid in einer Dosierung von $0,5-10(-20) \mu \mathrm{g} / \mathrm{kg} / \mathrm{min}$ unter intensivmedizinischer Überwachung notwendig.

C Über dem Pulmonalisareal ist im hypoxämischen Anfall oft kein Strömungsgeräusch auskultierbar.

D Neben der Beugung der Knie an die Brust kann eine großzügige Volumengabe oder Sedierung mit Morphin den Anfall durchbrechen.

E Zur Anfallsprophylaxe kann eine medikamentöse Therapie mit ß-Blockern wirksam sein. 


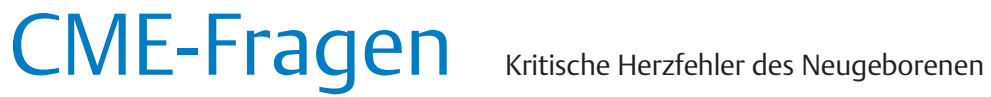

\section{8}

Welche Aussage zur Hämodynamik eines kritischen Herzfehlers ist zutreffend?
A Bei einer Pulmonalatresie mit intaktem Ventrikelseptum entspricht die Hämodynamik einer Extremvariante der Fallot-Tetralogie mit einer funktionellen Atresie der Pulmonalklappe.

B Der Grad der Obstruktion des linksventrikulären Ausflusstrakts bestimmt bei einer FallotTetralogie den intrakardialen Rechts-Links-Shunt und damit die Ausprägung der Zyanose.

C Die resultierende systemarterielle Sättigung wird bei einer Trikuspidalatresie durch verschiedene Faktoren beeinflusst, wie die Dimension eines Ventrikelseptumdefekts oder dem Ursprung der großen Arterien aus den Ventrikeln.

D Die Existenz von „major aortopulmonary collateral arteries“ (MAPCAs), über die die Lunge direkt aus der Aorta perfundiert wird, ist für eine Pulmonalatresie mit Ventrikelseptumdefekt ungewöhnlich.

E Bei einem hypoplastischen Linksherzsyndrom fließt das oxygenierte Blut über einen Rechts-LinksShunt aus dem rechten in den linken Vorhof.
Welche Angabe trifft bei einer simple d-Transposition der großen Arterien (d-TGA) nicht zu?
A Temporär kann es postnatal zu einer höheren post- als präduktalen Sättigung kommen. Hierdurch könnten diese Neugeborenen im postnatalen Pulsoxymetriescrenning übersehen werden.

B Gründe für das Nichtansteigen der systemarteriellen Sättigung nach erfolgreichem RashkindManöver können eine metabolische Azidose, eine linksventrikuläre Ausflussbahnobstruktion oder auch ein erhöhter Lungengefäßwiderstand sein.

C Der Blutfluss über den offenen Ductus arterious füllt den linken Vorhof und führt bei nicht restriktivem Vorhofseptum zu einer verbesserten Mischung auf Vorhofebene.

D Bei einem „good mixer“ kann es zu einer shuntbedingten Überdurchblutung der Lunge mit nachfolgender Herzinsuffizienz kommen.

E Neben einer ventrikuloarteriellen Diskordanz liegen bei einer simple d-TGA auch eine viszeroatriale und eine atrioventrikuläre Diskordanz vor.

\section{0}

Welche Aussage zu den Therapien bei kritischen Herzfehlern trifft nicht zu?
A Bei einer Pulmonalatresie mit intaktem Ventrikelseptum bestimmen neben der Morphologie des rechten Ventrikels auch die Koronarversorgung und die Größe der Trikuspidalklappe das weitere therapeutische Vorgehen.

B Eine arterielle Switch-OP sollte bei einer simple d-TGA innerhalb der 1. -2. Lebenswoche durchgeführt werden, da ansonsten der linke Ventrikel durch den physiologischen Abfall des Lungengefäßwiderstands an Muskelmasse verliert und postoperativ die Pumpleistung für den Systemkreislauf nicht mehr erbringen kann.

C Der „Hybridansatz“ ist bei Neugeborenen mit einem hypoplastischen Linksherzsyndrom ein alternatives Therapieverfahren zur Norwood-Operation. Es besteht aus einem chirurgischen Banding beider Pulmonalarterien und der katheterinterventionellen Implantation eines Duktusstents.

D Bei kurzstreckiger Aortenisthmusstenose kann eine Resektion der Engstelle mit anschließender angeschrägter „extended“ End-zu-End-Anastomose über eine linkslaterale Thorakotomie ohne Herz-Lungen-Maschine erfolgen.

E Bei Säuglingen mit einer Fallot-Tetralogie ist nach dem 5. Rezidiv eines hypoxämischen Anfalls die Indikation zur operativen Korrektur oder katheterinterventionellen Palliation gegeben. 